\title{
WILEY-VCH
}

DOI: 10.1002/admt.201700073

Article type: Full Paper

\section{Stretchable Electronic Platform for Soft and Smart Contact Lens Applications}

Andrés Vásquez Quintero*, Rik Verplancke, Herbert De Smet and Jan Vanfleteren

Dr. A. Vásquez Quintero

Centre for Microsystems Technology (CMST), imec and Ghent University, Technologiepark 15, 9052 Gent, Belgium

E-mail: andres.vasquez@ugent.be

Dr. R. Verplancke

Centre for Microsystems Technology (CMST), imec and Ghent University, Technologiepark 15, 9052 Gent, Belgium

Prof. Dr. H. De Smet

Centre for Microsystems Technology (CMST), imec and Ghent University, Technologiepark 15, 9052 Gent, Belgium

Prof. Dr. Jan Vanfleteren

Centre for Microsystems Technology (CMST), imec and Ghent University, Technologiepark 15, 9052 Gent, Belgium

Keywords: thermoplastic polyurethane (TPU); thermoforming; viscoelastic modelling; stretchable electronics; smart contact lens

A stretchable platform with spherical-shaped electronics based on thermoplastic polyurethane (TPU) is introduced for soft smart contact lenses. The low glass transition temperature of TPU, its relatively low hardness and its proven biocompatibility (i.e. protection of exterior body wounds) fulfill the essential requirements for eye wearable devices. These requirements include optical transparency, conformal fitting and flexibility comparable with soft contact lenses (e.g. hydrogel-based). Moreover, the viscoelastic nature of TPU allows planar structures to be thermoformed into spherical-caps with a well-defined curvature (i.e. eye's curvature at the cornea: $9 \mathrm{~mm}$ ). Numerical modeling and experimental validation enable finetuning of the thermoforming parameters and the optimization of strain-release patterns. Such tight control has been proven necessary to achieve oxygen permeable, thin, non-developable and wrinkle-free contact lenses with integrated electronics (silicon die, RF antenna and stretchable thin-film interconnections). This work paves the way towards fully autonomous smart contact lenses potentially for vision correction or sensing applications, among others. 


\section{WILEY-VCH}

\section{Introduction}

Recent advances in wearable electronics are not only focusing on devices integrated on textiles, ${ }^{[1]}$ footwear ${ }^{[2]}$ and accessories (i.e. head-bands, gloves, etc.) ${ }^{[3]}$ but also on conformal devices in close contact with the human body. ${ }^{[4]}$ The potential applications include the development of smart tattoos in contact with the skin, ${ }^{[5]}$ smart patches to monitor the status of skin wounds ${ }^{[6]}$ and smart contact lenses. ${ }^{[7]}$ The latter is foreseen to be applied in different fields ranging from biomedical diagnosis, ${ }^{[8-11]}$ signaling, ${ }^{[12]}$ and entertainment ${ }^{[13,14]}$ to vision correction (i.e. presbyopia), ${ }^{[9,15-19]}$ among others. However, challenges lie ahead mostly regarding to their integration into fully autonomous systems (i.e. power and decision independent). Among others, soft smart contact lenses are required to be conformal with the eye, stretchable, flexible, biocompatible, oxygen permeable and optically transparent (certainly on the path of sight). ${ }^{[7]}$ Moreover, their fabrication process needs to be robust, reliable and if possible compatible with reproducible and high volume fabrication techniques. ${ }^{[7]}$ The forming of polymers, including polyethylene terephthalate $(\mathrm{PET})^{[12,13,20]}$ and parylene-C, ${ }^{[21,22]}$ has been introduced as a suitable process to shape smart contact lenses with electronics. Although PET-based platforms can be easily thermoformed at temperatures starting at $80^{\circ} \mathrm{C}$ (its glass transition temperature is $85^{\circ} \mathrm{C}$ ), the resulting spherical shape is relatively more rigid compared to conventional soft contact lenses mainly due to its hardness level (ASTM D75 Rockwell R117). ${ }^{[23]}$ Although the forming of parylene-C structures can be made by cast molding of an embedding polymer (i.e. hydrogels, silicones), ${ }^{[21]}$ the initial electronics platform would be flat hindering the subsequent alignment steps. Parylene-C based electronics platforms could be thermoformed as well (to have an initial curved platform), however, this process demands a high temperature and long duration process (i.e. 6 to 48 hours at 170 or $\left.200^{\circ} \mathrm{C}\right)^{[22]}$ because of its high molecular weight and crystallinity, which would be incompatible with industrial scalability of smart contact lenses. Additionally, hardness level of parylene-C is similar to the one of PET (ASTM D75 Rockwell R80). ${ }^{[24]}$ For 


\section{WILEY-VCH}

this work, we have selected the thermoplastic polyurethane (TPU) as main mechanical carrier thanks to the combination of rubber and thermoplastic properties such as low softening temperature, low hardness (ASTM D2240 Shore A 87), high stretchability and the possibility to bond to itself at relatively low temperatures (required to fully embed structures). In this paper, we introduce a comprehensive approach in which a planar structure based on TPU films is thermoformed into a predetermined spherical-cap platform for smart contact lens applications. The presented approach resulted in a flexible, oxygen permeable and wrinklefree platform with electronics components, thus circumventing the previously described challenges for soft contact lenses. Due to its proximity to the eye the TPU films are required to be biocompatible and if possible with high microbial resistance and water vapor permeation to lessen ocular infections. Ether-based TPU films, as opposed to ester-based TPU, are demonstrated to be biocompatible in accordance with high viability of human fibroblasts cells during $96 \mathrm{~h}$ in-vitro cytocompatibility studies. ${ }^{[25]}$ In addition, antimicrobial tests (E. coli bacteria) shown a moderated resistance higher than the negative control. ${ }^{[25]}$ Besides its biocompatibility, TPU was shown to offer advantages regarding both its thermal and mechanical properties. Its relatively low glass transition temperature (around $120^{\circ} \mathrm{C}$ ) allowed the embedding and thermoforming of electronics systems, while maintaining their integrity. Additionally, its intrinsic stretchability allowed the realization of miniaturized platforms with comparable tensile properties (elastic modulus, tensile stress) and hardness of conventional soft contact lenses. ${ }^{[26,27]}$ Such characteristics are desired to assure maximum comfort during daily use (i.e. removing/inserting from/to the eye) and reduce delamination issues.

Numerical viscoelastic models enabled the fine-tuning of the thermoforming process (temperature and time wise), and permitted the design and optimization of cut out patterns, which increased the stretchability of the platforms. The patterned platforms were proven to accommodate undesired deformation during thermoforming, reducing the compressive strain near the edge, and hence avoiding buckling even for free boundary conditions. Moreover, the 


\section{WILEY-VCH}

cut out perforations let oxygen to flow freely to the cornea region, which is indispensable to avoid tight lens syndrome ${ }^{[28]}$ and/or and dry eye ${ }^{[29]}$ (caused by deprivation of oxygen). The spherical-shaped platform, with embedded electronics (Si chip, Radio-Frequency RF antenna and thin-film interconnections), has a predetermined curvature, enhanced stretchability and is compatible with hydrogel-based contact lenses. The approach presented here paves the way towards fully autonomous smart contact lenses, which if properly combined with electrooptical components would correct the vision of more than 1 billion people (i.e. when treating presbyopia $\left.^{[30]}\right)$.

\section{Results and Discussion}

The stretchable platform is composed of spherical-shaped electronics embedded (top and bottom) within two thermoplastic polyurethane (TPU) films. Figure 1 summarizes the thermoformed and patterned platform with an outer diameter of $10 \mathrm{~mm}$ and a curvature radius of $9 \mathrm{~mm}$, hence compatible with soft contact lenses (mean curvature at the cornea between 7 $\mathrm{mm}$ and $9 \mathrm{~mm}) .{ }^{[31]}$ Figure 1a presents the details of the platform, indicating the form factor and location of the electronics components (silicon chip, RF antenna and thin-film interconnections), placed in respective polymeric semi-rigid islands. Silicon chips can potentially include combinations of modules with data processing/saving capabilities, sensor front-end, electro-optical cell driving and RF interfacing (both for communication and energy transfer). The latter is thought to be implemented in the high frequency range (at $13.56 \mathrm{MHz}$ ) with Near-Field Communication (NFC) protocols. The relatively good energy transmission rate of NFC occurs at short distances (when coupled magnetically), which hinders large range links and limits the data/energy transfer to be performed only in close proximity to the reader (e.g. NFC-enabled phone, custom-made reader, etc.). ${ }^{[32]}$ Ultra-compact loop NFC antennas are required due to the highly constricted space on the lens. The small dimensions (diameter around $10 \mathrm{~mm}$ ), needs to be compensated with a higher thickness to keep a representative quality factor. Fine pitch and thick loop antennas are thought to be achieved by pattern plating 


\section{WILEY-VCH}

of a thin metallic seed through a thick resist layer. ${ }^{[33]}$ Additionally, on-chip sensors can be designed to save space and increase integration yield, for instance, photodiodes to measure the light intensity of the environment. ${ }^{[34]}$ Besides silicon-based implementation of electronics, thin-film transistor driving circuits on polyimide can be integrated taking advantage of its similar mechanical properties (e.g. flexural rigidity). ${ }^{[34]}$ Active progress on planar organic field effect transistors used as biochemical sensors make them potential candidates to be integrated on a smart contact lens. ${ }^{[9,35]}$ Measured concentrations of analytes on tear fluid compared to blood provide several candidates to be monitored for diagnostic, for instance, lactate for the liver disease, urea for renal function and dopamine for glaucoma. ${ }^{[7]}$

The center region of the platform is reserved for the integration of an optical cell, foreseen for vision correction applications (e.g. to treat aniridia, presbyopia). ${ }^{[14,15]}$ The deformation from a 2D disk to a 3D spherical shape, including electronics, was performed by means of thermoforming (i.e. heating and shaping in a metallic mold) (Figure 1b). The TPU substrate lies at the core of the thermoforming process, thanks to its low softening temperature and viscoelastic nature. The mechanical properties of the TPU films are required to match as close as possible the ones of conventional soft contact lenses (e.g. hydrogel-based) in order to reduce delamination issues, and discomfort and irritation to the wearer. The TPU Platilon ${ }^{\circledR}$ U4201 AU from BAYER ${ }^{\odot}$ was of particular interest for this work thanks to its similar mechanical properties compared to soft lenses, mainly based on 2-Hydroxy-ethyl methacrylate (HEMA). For instance, TPU's elastic modulus is only five times higher (10 MPa versus $2 \mathrm{MPa}$ ) and their hardness are alike (Shore A87 versus Shore A85.6), ${ }^{[26,27]}$ whereas its maximum elongation is fifteen times higher $(550 \%$ versus $43 \%) .{ }^{[36]}$ Hence these akin properties assure the platform's mechanical integrity when subjected to tensile, shear and/or bending forces. This, in combination with the biocompatible medical uses of TPU Platilon ${ }^{\circledR}$ U4201 found in literature (from wound protection to biosensors), ${ }^{[37-39]}$ and supported by the 


\section{WILEY-VCH}

respective cytocompatibility and microbial tests ${ }^{[25]}$ validate its use as carrier for smart and soft contact lenses.

The thin-film interconnections consist of gold traces with top/bottom polyimide (PI) protective coatings to create a conformal encapsulation of only $11 \mu \mathrm{m}$ thick. The circuitry is made stretchable along a horizontal plane thanks to the meandered-interconnection design between flexible, non-stretchable and functional PI islands (Figure 1c). The islands are envisioned for the support and mechanical protection of Si chips and/or TFT based circuits, among others, which can be either rigid or flexible. ${ }^{[40,41]}$ The electrical interconnection between a silicon daisy chain chip (DCC) and the thin-film circuitry is achieved via a flipchip technique based on Laser-Induced Forward Transfer (LIFT) of bumps of indium (In). ${ }^{[42]}$ The Si chip was thinned down to $30 \mu \mathrm{m}$ of thickness in order to reduce enough its flexural rigidity $(D)$, and hence endure bending forces without breaking (Figure 1d). This is valid since the flexural rigidity is directly proportional to the thickness cubed as in: $D=E t^{3} / 12(1-$ $\left.v^{2}\right),{ }^{[43]}$ where $E, v$ and $t$ are the Young's modulus, Poisson ratio and thickness of the chip, respectively.

Tensile and compressive strains are inevitable during the thermoforming process since it involves a transformation of a developable surface (e.g. circular plane) into a non-developable or zero-Gaussian curvature (e.g. spherical cap). ${ }^{[44-46]}$ Relatively high compressive strain values are experienced by the TPU platforms along the outer edge due to their limited stretchability and free boundary conditions during the thermoforming (i.e. the convex-shaped mold pulls the platform to the center forcing a reduction in the disk perimeter). Consequently, this produces buckling and wrinkles which could hamper the electronic functionality of electronic components near the edge (e.g. Si chips, RF antennas). ${ }^{[15]}$ The strain profile of a full disk of radius $R$ along the hoop (or circumferential) direction is $\varepsilon_{h}=\sin (\theta) / \theta-1,{ }^{[47]}$ where $\theta=R / r$ is the ratio between the disk radius and its curvature radius ' $r$ '. This relationship holds valid between $0 \leq \theta \leq \pi$, and it is physically limited by the flexural strength of the deformed 


\section{WILEY-VCH}

material. For a non-patterned circular platform with a radius of $6.5 \mathrm{~mm}$ and a curvature radius of $8 \mathrm{~mm}$ (representative values for soft contact lenses), the maximum compressive strain is calculated to be around $-10 \%$ at the outer edge (Figure S1 Supporting Information). This level of compression is not tolerated without buckling by the gold RF antenna embedded in PI near the edge of the platform as exemplified in Figure 2.

The packing of a naturally flat thin elastic sheet into a spherical surface has been analytically described by the Elastica equation providing the buckle behavior and its periodicity. ${ }^{[46]}$ As experienced when covering a sphere with a flat sheet, the latter buckles in conical shapes with one or multiple folds. During the thermoforming of disks into sphericalcaps, the compression mold further restricts the folds, producing a higher fold periodicity with lower amplitude. This is observed in Figure 2a, where the RF antenna near the border of a thermoformed full disk presents periodical wrinkles. Fold formation when closing the mold was also predicted with numerical simulations, where high compressive and tensile strain values are present at their peaks and valleys. The simulation did not converge after a simulated time of $1 \mathrm{~s}$, due to the high fold instability.

Perforations along the surface of the TPU platform before thermoforming were proven to be necessary in order to avoid buckling formation and fabricate wrinkle-free spherical caps. Additionally, the perforations serve to duplicate more accurately the desired spherical shape (geometry of the mold) and allow oxygen flow, essential for soft smart contact lenses. Such perforations, called strain-release patterns, are cut out from the circular platforms which in turn give rise to the supporting mechanical meanders between the outer and inner rings of the platform. These meanders enhance the platform stretchability by accommodating deformation along the radial direction during thermoforming and daily use. Moreover, the platform relaxes the built-up stress thanks to the TPU's viscoelastic nature. Straight supports between the inner and outer rings restrict the buckling only at their base, with very localized but high compressive and tensile strain (Figure 2b). Additionally, the initial circular shape of the inner 


\section{WILEY-VCH}

and outer rings is distorted by the straight supports pulling towards the center during thermoforming. However, the use of meandered-shapes for the supports clearly reduces buckling to the point of avoiding wrinkles (Figure 2c). The principal strain along the hoop direction is more evenly distributed, thanks to the deformation of the fine-tuned TPU-based meanders, as shown in the results of numerical simulations in Figure 2.

Figure 3 shows the validated simulation results of the mechanical analysis during the thermoforming process for the TPU platform, regarding its curvature, maximum sag (lens height), and strain/stress values. Figure 3 a indicates a maximum vertical displacement of 3 $\mathrm{mm}$ at the center region of the platform, and the direction of the radial strain accommodated by the strain-release pattern. The thermoforming simulations were developed with COMSOL Multiphysics in the $3 \mathrm{D}$ space $\left(180^{\circ}\right.$ symmetry - Figure $\left.1 \mathrm{~b}\right)$ with mechanical boundary conditions of friction between the mold and TPU, and free elsewhere. The TPU was modeled by implementing the Generalized Maxwell viscoelastic model (Equation 1$)^{[48]}$ in combination with the William-Landel-Ferry thermal shift function. ${ }^{[49]}$

$$
\sigma(t)=\epsilon \cdot \sum_{1}^{n} E_{i} \cdot \exp \left(\frac{-t}{\tau_{i}}\right)
$$

Where, $\sigma(t)$ is the stress dependent on time, $\epsilon$ is the stretch value, $E$ is the Young's modulus, $\tau$ is the relaxation time and $n$ is the number of coefficients of the series, respectively.

Experimental data of the geometry and strain distribution are in good agreement with the numerical modeling. The relative difference between the measured and simulated curvatures after the thermoforming was proven to be lower than $5 \%,\left(\left(\kappa_{\mathrm{SIM}}-\kappa_{\mathrm{MEAS}}\right) / \kappa_{\mathrm{MEAS}}\right) * 100 \%$, where $\kappa_{\mathrm{MEAS}}$ and $\kappa_{\mathrm{SIM}}$ are the measured and simulated curvature values, respectively. It is also noted that the final curvature of the platform is bigger for a lower processing temperature, always above the curvature of the mold due to the spring-back of the TPU. The curvature as well as the ' $z$ ' profiles of the platforms are represented in Figure $3 \mathrm{~b}$, where $R=0 \mathrm{~mm}$ corresponds to the center of the platform. The details of the model as well as its validation by 


\section{WILEY-VCH}

experimentation are presented in Figure S2 and Figure S3 (Supporting Information), respectively. The complete curvature results at different processing temperatures and time steps are shown in Figure S4 (Supporting Information).

Figure 3c,d shows the 3D representation of the principal stress and strain of the platform throughout the thermoforming steps, which consist in closing the mold (between 0 and $5 \mathrm{~s}$ ) at a rate of $20 \mathrm{~mm} \mathrm{~min}^{-1}$ to prevent tensile strain hardening ${ }^{[50]}$, keeping the mold closed (between $5 \mathrm{~s}$ and $60 \mathrm{~s}$ ) and demolding (between $60 \mathrm{~s}$ and $65 \mathrm{~s}$ ). The forming temperature (i.e. $80^{\circ} \mathrm{C}, 120^{\circ} \mathrm{C}$ and $140^{\circ} \mathrm{C}$ ) is kept constant from $0 \mathrm{~s}$ to $30 \mathrm{~s}$, and it is brought back to room temperature between $30 \mathrm{~s}$ and 50s. The principal stress values (Figure $3 \mathrm{c}$ ) are maximum (-20 $\mathrm{MPa}$ ) at $5 \mathrm{~s}$ around the DCC region. This region is virtually stress-free at $60 \mathrm{~s}$ thanks to the viscoelastic relaxation nature of the TPU, thus increasing the platform's mechanical robustness. The principal strain values (Figure 3d) remain the same during the relaxation period ( $5 \mathrm{~s}$ to $60 \mathrm{~s}$ ), due to the mechanical constriction provided by the shape of the mold. Although the maximum tensile deformation occurs at the supporting meanders (around 3.5\%), this value is well below the maximum strain limit of TPU $(>500 \%) .{ }^{[36]}$ Strain relaxation occurred during the demolding process ( $60 \mathrm{~s}$ to $65 \mathrm{~s}$ ), generating a mechanically stable platform with a radius of curvature of $9 \mathrm{~mm}$.

Figure 4 summarizes the results of the mechanical analysis of the thin-film circuitry and the DCC chip. Meandered conductive interconnections were designed between rigid PI islands with components, in order to allow for in-plane deformations. Figure 4a shows a picture of the thin-film circuitry embedded on flat TPU sheets before patterning and thermoforming. Simulation results confirmed the change in geometry of the thin-film circuitry from a flat to a spherically shaped structure (Figure $4 b$ ). It was found that the final location of the thin-film circuity and DCC chip strongly depends on the thermoforming temperature, Figure S5 (Supporting Information). For a given higher temperature a higher TPU deformation is expected, since the platform's surface is closer to the spherical shape of the 


\section{WILEY-VCH}

mold. Figure 4c,d shows the 3D representation of the maximum principal stress and strain of the DCC and interconnection meanders when the mold is closed. Although the electrical meanders accommodate elongations along the principal hoop direction, localized tensile strain of maximum $1.5 \%$ occurred in the PI island around the DCC chip. This strain, however, is reduced by the viscoelastic relaxation during the thermoforming. The maximum principal stress is located at the DCC with values around -100 MPa, which is well below the Si fracture strength $\left(3.5 \mathrm{GPa}^{[51]}\right)$. The mechanical analysis of meanders with smaller apertures shows the same order of magnitude for the generated stress and strain distributions, Figure S6 (Supporting Information).

Due to the high miniaturization level of the platforms, electrical interconnections at relatively small dimensions (pitch and thickness) are essential to integrate electronics components. This is achieved with the LIFT of In bumps directly onto the gold thin-film circuitry (Figure 4e), which resulted in very thin and homogenous interconnections, Figure S7 (Supporting Information) Indium bumps on gold are advantageous thanks to their low melting temperature $\left(\sim 120^{\circ} \mathrm{C}\right)$, thereby facilitating the integration. In addition, the $\mathrm{Au} / \mathrm{In}$ intermetallic compounds formed during this low temperature process remain stable up to $450{ }^{\circ} \mathrm{C},{ }^{[52]}$ thus not degrading during subsequent high temperature processes (i.e. thermoforming and/or lamination). The DCC was bonded at $120^{\circ} \mathrm{C}$ for 5 minutes, which proved to be sufficient to obtain a stable and ohmic interconnection resistance (Figure 4f). Measured via resistances increased around 5\% after the thermoforming step and no difference was observed on scanning electron microscopy images.

Mechanical robustness against bending forces, typically exerted when inserting/removing the lens from the eye, was assessed by static and dynamic flexion tests along two different axis (i.e. $A$ axis along the DCC chip and $B$ axis perpendicular to the first one). Figure 5a shows the relative change in resistance at a fixed distance between two parallel plates (compressing the platform), from $10 \mathrm{~mm}$ to $1 \mathrm{~mm}$ (three samples per condition). The inserts 


\section{WILEY-VCH}

show the platforms being deformed along the A and B axis, at different distances, respectively. On one hand, the change in resistance is bigger when the deformation occurred along the DCC, reaching $8 \%$ at $1 \mathrm{~mm}$. On the other hand, relative changes in resistance were below $1 \%$ at $1 \mathrm{~mm}$ when the deformation occurred in the perpendicular axis. Figure $5 \mathrm{~b}$ shows the dynamic test up to 100 cycles at a distance of $3.5 \mathrm{~mm}$ between the parallel plates. Although the trends lie in similar ranges of variation, deformation along the A axis is proven to have a bigger effect on the vias resistance. This bigger change is thought to be caused by the bending of the PI island, where the DCC is placed, being the precursor of delamination of the In-based bump.

Figure 6 presents images of the stretchable platform attached by means of van der Waals forces to a commercial hydrogel-based 1-day contact lens in order to suggest scenarios of real applications. Figure $6 \mathrm{a}, \mathrm{b}$ shows the stack placed on finger tips mimicking the actions of inserting and removing the contact lens from the user's eye. Even though during the insertion action, the lens experiences low bending forces, the removal action exerts small bending radii as shown in Figure 6b. Nevertheless, thanks to its flexibility and shape memory, it is able to withstand such bending forces and return to its thermoformed shape. Figure $6 \mathrm{c}$ shows the platform/contact lens stack in a container within a saline solution $(0.9 \%$ saline $)$ at room temperature used to wash and keep clean commercial contact lenses. Several samples were immersed in the solution for period up to 16 days, where the vias resistance of the DCC was measured every 3 days (platforms were removed, DI washed, dried and measured). Figure 6d shows the measured resistance and relative change in resistance for the aforementioned tests. It is noted that even though the resistance increases with time it reaches a relative change of $5.6 \%$ after 16 days of exposure. The resistances of the vias were calculated from V/I curves measured after the respective exposures, as shown in Figure S11 (Supporting Information).

\section{Conclusion}




\section{WILEY-VCH}

A comprehensive approach including materials, design, integration techniques and thermoforming methods is here introduced to realize stretchable platforms based on TPU with spherical-shaped electronics for smart contact lenses. TPU intrinsic mechanical properties match the ones of soft contact lenses, thus reducing risks of interface delamination and increasing the comfort for the wearer. Precise thermoforming steps constitute the base for deforming 2D planar surfaces into 3D curvilinear and non-developable structures.

Miniaturized compact geometries combined with optimized strain-release patterns enhanced the stretchability nature of the TPU resulting in wrinkle-free, extremely flexible, and even oxygen transparent platforms. Furthermore, thin-film circuits with silicon chips embedded on TPU substrates allows for a reliable fabrication of smart contact lenses with a predetermined curvature and ready to be inserted into materials compatible with the eye (e.g. hydrogels and silicones). The concepts presented here are applicable to realize specific platforms with a determined number and type of components (e.g. wireless systems and actuators) and for diverse close-to-the-body healthcare diagnose and monitoring scenarios.

\section{Experimental Section}

Fabrication of thin-film circuitry: A first layer of polyimide $(5.5 \mu \mathrm{m}$ thick, PI-2611, HD Microsystems) spin-coated on a previously cleaned glass wafer at $3000 \mathrm{rpm}$ for $30 \mathrm{~s}$ (ramp: 5 $\mathrm{s}$ at $500 \mathrm{rpm}$ ), dried on a hot plate at $200{ }^{\circ} \mathrm{C}$ for $10 \mathrm{~min}$, and cured in a nitrogen oven at $350{ }^{\circ} \mathrm{C}$ for $60 \mathrm{~min}$ (profile: from RT to $200{ }^{\circ} \mathrm{C}$ at $4{ }^{\circ} \mathrm{C} \mathrm{min}^{-1} ; 30 \mathrm{~min}$ at $200{ }^{\circ} \mathrm{C}$; from $200{ }^{\circ} \mathrm{C}$

to $350{ }^{\circ} \mathrm{C}$ at $2.5^{\circ} \mathrm{C} \mathrm{min}^{-1}$ ) formed the bottom encapsulation of the circuitry. A sputtered thinfilm of gold $\mathrm{Au}$ (150 nm thick) with an adhesion enhancement layer of TiW (50 nm thick; $10 \% / 90 \%$ ) served as the conductor of the deformable circuitry. The metallic layer was patterned by photolithography (S1818 photoresist, spin-coating at $4000 \mathrm{rpm}$ for $60 \mathrm{~s}$, baking at $90{ }^{\circ} \mathrm{C}$ for $2 \mathrm{~min}$, UV irradiance for $85 \mathrm{~mJ} \mathrm{~cm}{ }^{2}$, developing for $30 \mathrm{~s}$ with developer MF-319 / deionized water (DI) solution of 1:1 volume ratio), and wet etching (Au: solution 1g $\mathrm{I}_{2}, 4 \mathrm{~g}$ $\mathrm{KI}$ and $73 \mathrm{ml} \mathrm{H} \mathrm{H}_{2} \mathrm{O}$ for $2 \mathrm{~min}$; TiW: solution $\mathrm{H}_{2} \mathrm{O}_{2}$ at $55^{\circ} \mathrm{C}$ for $20 \mathrm{~s}$ with 2 min rinsing in DI 


\section{WILEY-VCH}

water). A second $5.5 \mu \mathrm{m}$ thick coating of PI spin-coated in the same conditions as the bottom encapsulation covered the Au circuitry to form the top side finish. A sputtered thin-film of Al (200 nm thick) with an adhesion enhancement layer of TiW (20 nm thick; 10\%/90\%) patterned by photolithography (S1818 photoresist with the same parameters as before), and wet etching (Al: solution $\mathrm{CH}_{3} \mathrm{COOH}: \mathrm{H}_{3} \mathrm{PO}_{4}: \mathrm{HNO}_{3}$ :DI; volume ratios: (4(> 90\%):4(> 89\%):1(> 65\%):1(100\%)) for $10 \mathrm{~min}$; TiW: same as before) served as a hard mask to pattern the PI surrounding the Au circuitry and to create via holes through the exposed top PI cover layer using RIE etching (150 mTorr, $15 \mathrm{sccm} \mathrm{O}_{2}, 5 \mathrm{sccm} \mathrm{CHF}_{3}$ ). The thin-film of $\mathrm{Au}$ at the location of the pads served as the RIE stop layer. The RIE power ranged between $150 \mathrm{~W}$ and $40 \mathrm{~W}$ in order to optimize the surface and electrical properties of the Au pads at the openings, as shown in Figure S8 (Supporting Information). The measured PI etching rate for $150 \mathrm{~W}, 70$ $\mathrm{W}, 60 \mathrm{~W}$, and $40 \mathrm{~W}$ are: $0.27 \mu \mathrm{m} \mathrm{min}^{-1}, 0.20 \mu \mathrm{m} \mathrm{min}^{-1}, 0.18 \mu \mathrm{m} \mathrm{min}^{-1}$ and $0.11 \mu \mathrm{m} \mathrm{min}{ }^{-1}$, respectively, which determined the etching duration for $11 \mu \mathrm{m}$ thick PI as: $40.7 \mathrm{~min}, 54.8 \mathrm{~min}$, $60.4 \mathrm{~min}$ and $110.5 \mathrm{~min}$, respectively. As an alternative process, a second Al hard mask (deposited and etched as before) placed at the location of the contact pads protected the $\mathrm{Au}$ pads from the first RIE step (durations for $11 \mu \mathrm{m}$ thick PI), while a second RIE step was set to etch the top $5.5 \mu \mathrm{m}$ thick PI layer at $40 \mathrm{~W}$ (duration of $50.3 \mathrm{~min}$ ). Via resistance at different surface roughness (e.g. $\mathrm{R}_{\mathrm{a}}=2.83 \mathrm{~nm}$ at $70 \mathrm{~W}$ and $\mathrm{R}_{\mathrm{a}}=1.40 \mathrm{~nm}$ at $40 \mathrm{~W}$, shown in Figure $\mathrm{S} 8$ ) were similar within standard deviation of three samples. Al and TiW etchants removed the second hard mask from the platform surface. The thin-film process flow and further steps are illustrated in Figure S9 (Supporting Information).

LIFT and flip-chip integration: A pico-second laser (355 nm, $12 \mathrm{ps)} \mathrm{from} \mathrm{3D} \mathrm{Micromac}$ operated with a fluence of $\sim 270 \mathrm{~mJ} \mathrm{~cm}^{-2}$ transferred the In bumps from a 4 inch glass wafer (donor: $200 \mathrm{~nm}$ thick evaporated In film) directly to the Au pads of the platform (receiver) when in contact using the Laser-Induced Forward Transfer (LIFT) technique. ${ }^{[42]}$ Three single pulses served to transfer In bumps which are $10 \mu \mathrm{m}$ wide and $7 \mu \mathrm{m}$ thick (after each pulse the 


\section{WILEY-VCH}

donor was moved to a new area). Electroless deposition of $\mathrm{Ni} / \mathrm{Au}$ bumps (30 $\mathrm{min}$ in Melplate Ni8600 UK solution $+8 \mathrm{~min}$ in Aurolectroless STM solution) onto the aluminum pads of the DCC resulted in a thickness of $15 \mu \mathrm{m}$, as shown in Figure S10 (Supporting Information). All chips were thinned down $(<30 \mu \mathrm{m}$ thick) with the Logitech PM5 Lapping and Polishing machine (10 rpm with control over the thickness every $3 \mu \mathrm{m})$, and integrated in bare die format on the deformable PI-based circuitry. Using a Dr. Tresky flip-chip semi-automatic machine, the DCC's were aligned and bonded with a pressure of $130 \mathrm{k} \mathrm{Pa}\left(5 \mathrm{~min}\right.$ at $\left.150{ }^{\circ} \mathrm{C}\right)$. Lamination and thermoforming: A vacuum wafer bonder (Logitech WSB2) allowed the simultaneous lamination (soaking time: $2 \mathrm{~min}$, bonding time: $10 \mathrm{~min}$ at $120^{\circ} \mathrm{C}$ and $1.5 \mathrm{bar}$ ) of a $100 \mu \mathrm{m}$ thick medical grade thermoplastic polyurethane (TPU: Platilon ${ }^{\circledR}$ U4201 AU from BAYER(C) at the top and bottom sides of the platform. A pico-second laser (355 nm, $12 \mathrm{ps,}$ $50 \mathrm{kHz}, 320 \mathrm{~mW}$ ) ablated the double-sided TPU encapsulation using five passes at $20 \mathrm{~mm} \mathrm{~s}^{-1}$, in order to create the strain-release pattern and to increase the oxygen flow of the platform. The thermoforming step $\left(5 \mathrm{~min}\right.$ at $\left.120^{\circ} \mathrm{C}\right)$ used a metallic mold with spherical surfaces with convex and concave radii of curvature of $8 \mathrm{~mm}$ and $8.2 \mathrm{~mm}$, respectively (the gap of $200 \mu \mathrm{m}$ corresponds to the thickness of the deformable platform to prevent squeezing it).

Electrical characterization: Electrical characterization used a configurable Keithley 6220 current source and a Keithley 2182A nanovoltmeter in combination with a probe station which allowed for 4-points resistance measurement. The current source generated a current from $-1 \mathrm{~mA}$ to $1 \mathrm{~mA}$ which in return generated a voltage drop in the order of $\mathrm{mV}$ when passing through the DCC's vias and the thin-film track resistance, as shown in Figure S10 (Supporting Information). The 4-points resistance measurement was used to assess the electrical functionality of the platform, before and after thermoforming process, during the saline immersion test and the static and dynamic bending tests.

\section{Supporting Information}




\section{WILEY-VCH}

Supporting Information is available from the Wiley Online Library or from the author.

\section{Acknowledgements}

This work was funded by the Early PostDoc Fellowship from the Swiss National Science Foundation (SNSF), under the grant No. 158871, and the Marie Curie IntraEuropean Fellowship No. 661092. The authors thank the support team at CMST/imec.

Received: ((will be filled in by the editorial staff))

Revised: ((will be filled in by the editorial staff))

Published online: ((will be filled in by the editorial staff))

[1] D. De Rossi, Nat Mater 2007, 6, 328.

[2] Stretchable Electronics; Someya, T., Ed.; Wiley-VCH Verlag GmbH \& Co. KGaA:

Weinheim, Germany, 2009.

[3] M. Stoppa, A. Chiolerio, Wearable Electronics and Smart Textiles: A Critical Review.

Sensors 2014, 14.

[4] J.-H. Ahn, J. H. Je, J. Phys. D. Appl. Phys. 2012, 45, 103001.

[5] D.-H. Kim, N. Lu, R. Ma, Y.-S. Kim, R.-H. Kim, S. Wang, J. Wu, S. M. Won, H. Tao,

A. Islam, K. J. Yu, T. Kim, R. Chowdhury, M. Ying, L. Xu, M. Li, H.-J. Chung, H.

Keum, M. McCormick, P. Liu, Y.-W. Zhang, F. G. Omenetto, Y. Huang, T. Coleman,

J. A. Rogers, Science (80-. ). 2011, 333, 838.

[6] S. L. Swisher, M. C. Lin, A. Liao, E. J. Leeflang, Y. Khan, F. J. Pavinatto, K. Mann, A. Naujokas, D. Young, S. Roy, M. R. Harrison, A. C. Arias, V. Subramanian, M. M.

Maharbiz, Nat. Commun. 2015, 6, 6575.

[7] N. M. Farandos, A. K. Yetisen, M. J. Monteiro, C. R. Lowe, S. H. Yun, Adv. Healthc. Mater. 2015, 4, 792.

[8] C. Alvarez-Lorenzo, H. Hiratani, A. Concheiro, Am. J. Drug Deliv. 2006, 4, 131.

[9] M. Senior, Nat Biotech 2014, 32, 856.

[10] K. Mansouri, T. Shaarawy, Br. J. Ophthalmol. 2011, 95, 627.

[11] M. Leonardi, E. M. Pitchon, A. Bertsch, P. Renaud, A. Mermoud, Acta Ophthalmol. 


\section{WILEY-VCH}

2009, $87,433$.

[12] J. Pandey, Yu-Te Liao, A. Lingley, R. Mirjalili, B. Parviz, B. Otis, IEEE Trans. Biomed. Circuits Syst. 2010, 4, 454.

[13] H. Ho, E. Saeedi, S. S. Kim, T. T. Shen, B. A. Parviz, Proc. IEEE Int. Conf. Micro Electro Mech. Syst. 2008, 403.

[14] J. De Smet, A. Avci, P. Joshi, D. Schaubroeck, D. Cuypers, H. De Smet, J. Soc. Inf. Disp. 2013, 21, 399.

[15] J. De Smet, A. Avci, R. Beernaert, D. Cuypers, H. De Smet, J. Disp. Technol. 2012, 8, 299.

[16] F. Carpi, G. Frediani, S. Turco, R. D. De, Adv. Funct. Mater. 2011, 21, 4152.

[17] S. J. Woltman, G. D. Jay, G. P. Crawford, Nat Mater 2007, 6, 929.

[18] J. De Smet, P. De Backer, I. E., P. Joshi, D. Cuypers, H. De Smet, In EuroDisplay 2013; 2013; pp. 61-64.

[19] H. De Smet, J. De Smet, P. De Backer, P. Joshi, E. Islamaj, X. Shang, D. Cuypers, In Society for Information Display, Mid-Europe Chapter, Spring Meeting; 2014; p. 22.

[20] J. De Smet, A. Avci, R. Beernaert, D. Cuypers, H. De Smet, J. De Smet, J. Disp. Technol. 2012, 8, 299.

[21] J.-C. Chiou, S.-H. Hsu, Y.-C. Huang, G.-T. Yeh, W.-T. Liou, C.-K. Kuei, Sensors 2017, $17,108$.

[22] B. J. Kim, B. Chen, M. Gupta, E. Meng, J. Micromechanics Microengineering 2014, $24,65003$.

[23] J. R. Cahoon, W. H. Broughton, A. R. Kutzak, Metall. Trans. 1971, 2, 1979.

[24] J. C. Lötters, W. Olthuis, P. H. Veltink, P. Bergveld, J. Micromechanics Microengineering 1999, 7, 145.

[25] A. Alves, R. Cardoso, T. Correia, B. Antunes, I. Correia, P. Ferreira, Colloids Surfaces B Biointerfaces 2014, 113, 25. 


\section{WILEY-VCH}

[26] I. Tranoudis, N. Efron, Contact Lens Anterior Eye 2004, 27, 177.

[27] T. Löher, A. Ostmann, G. M. Allee, M. Seckel, In CMPT Symposium Japan (ICSJ); 2014; pp. 177-181.

[28] P. Netland, CLAO J. 1990, 16, 308.

[29] C. G. Begley, B. Caffery, K. K. Nichols, R. Chalmers, Optom. Vis. Sci. 2000, 77.

[30] B. A. Holden, T. R. Fricke, S. M. Ho, R. Wong, G. Schlenther, S. Cronjé, A. Burnett, E. Papas, K. S. Naidoo, K. D. Frick, Arch. Ophthalmol. 2008, 126, 1731.

[31] T. Olsen, Br. J. Ophthalmol. 1986, 70, 152.

[32] J. Kim, A. Banks, Z. Xie, S. Y. Heo, P. Gutruf, J. W. Lee, S. Xu, K. I. Jang, F. Liu, G. Brown, J. Choi, J. H. Kim, X. Feng, Y. Huang, U. Paik, J. A. Rogers, Adv. Funct. Mater. 2015, 25, 4761 .

[33] B. Loechel, J. Micromechanics Microengineering 2000, 10, 108.

[34] F. De Roose, S. Steudel, K. Myny, M. Willegems, S. Smout, M. Ameys, P. Malinowski, R. Gehlhaar, R. Poduval, X. Chen, J. De Smet, A. Vásquez Quintero, H. De Smet, W. Dehaene, J. Genoe, In IEDM 2016; 2016; pp. 798-801.

[35] B. Yaman, I. Terkesli, K. M. Turksoy, A. Sanyal, S. Mutlu, Org. Electron. physics, Mater. Appl. 2014, 15, 646.

[36] R. A. Barb, B. Magnus, S. Innerbichler, T. Greunz, M. Wiesbauer, R. Marksteiner, D. Stifter, J. Heitz, Appl. Surf. Sci. 2015, 325, 105.

[37] H. Sillanpää, E. Halonen, T. Liimatta, M. Mäntysalo, In 2014 International Conference on Electronics Packaging, ICEP 2014; 2014; pp. 322-325.

[38] J. Tolvanen, J. Hannu, J. Palosaari, M. Nelo, H. Jantunen, Electron. Lett. 2013, 52, 1395.

[39] C. Koppenborg, Kunststoffe Int. 2011, 5, 58.

[40] R. Carta, P. Jourand, B. Hermans, J. Thoné, D. Brosteaux, T. Vervust, F. Bossuyt, F. Axisa, J. Vanfleteren, R. Puers, Sensors Actuators, A Phys. 2009, 156, 79. 


\section{WILEY-VCH}

[41] S. P. Lacour, J. Jones, S. Wagner, T. Li, Z. Suo, Proc. IEEE 2005, 93, 1459.

[42] K. S. Kaur, J. Missinne, G. Van Steenberge, Appl. Phys. Lett. 2014, 104, 61102.

[43] A. V. Quintero, D. Briand, N. F. de Rooij, J. Micromechanics Microengineering 2015, $25,45013$.

[44] J. A. Rogers, T. Someya, Y. Huang, Science 2010, 327, 1603.

[45] H. C. Ko, M. P. Stoykovich, J. Song, V. Malyarchuk, W. M. Choi, C.-J. Yu, J. B. Geddes III, J. Xiao, S. Wang, Y. Huang, J. A. Rogers, Nature 2008, 454, 748.

[46] E. Cerda, L. Mahadevan, Proc. R. Soc. A Math. Phys. Eng. Sci. 2005, 461, 671 LP.

[47] R. Dinyari, S. B. Rim, K. Huang, P. B. Catrysse, P. Peumans, Appl. Phys. Lett. 2008, $92,1$.

[48] S. Lan, H. J. Lee, S. H. Lee, J. Ni, X. Lai, H. W. Lee, J. H. Song, M. G. Lee, Mater. Des. 2009, 30, 3879.

[49] M. L. Williams, R. F. Landel, J. D. Ferry, J. Am. Chem. Soc. 1955, 77, 3701.

[50] Y. M. Luo, L. Chevalier, E. Monteiro, Int. J. Mater. Form. 2014, 7, 359.

[51] J. Bagdahn, W. N. Sharpe, J. Microelectromechanical Syst. 2003, 12, 302.

[52] T.-T. Luu, N. Hoivik, K. Wang, K. E. Aasmundtveit, A.-S. B. Vardøy, Metall. Mater. Trans. A 2015, 46, 2637.

[53] G. Lu, D. M. Kalyon, Polym. Eng. Sci. 2003, 43, 1863.

[54] S. M. Perinchery, E. C. P. Smits, a Sridhar, P. Albert, J. van den Brand, R. Mandamparambil, I. Yakimets, H. F. M. Schoo, Laser Phys. 2014, 24, 66101.

[55] Y. Hsu, M. Gonzalez, F. Bossuyt, J. Vanfleteren, I. De Wolf, S. Member, 2011, 58, 2680.

[56] R. Verplancke, F. Bossuyt, D. Cuypers, J. Vanfleteren, J. Micromechanics Microengineering 2012, 22, 15002.

[57] M. Gonzalez, F. Axisa, M. Vanden Bulcke, D. Brosteaux, B. Vandevelde, J. Vanfleteren, Microelectron. Reliab. 2008, 48, 825. 


\section{WILEY-VCH}

[58] D. S. Gray, J. Tien, C. S. Chen, Adv. Mater. 2004, 16, 393.

[59] Y. Hsu, M. Gonzalez, F. Bossuyt, F. Axisa, J. Vanfleteren, I. De Wolf, J. Van, I. De Wolf, Thin Solid Films 2011, 519, 2225. 


\section{WILEY-VCH}

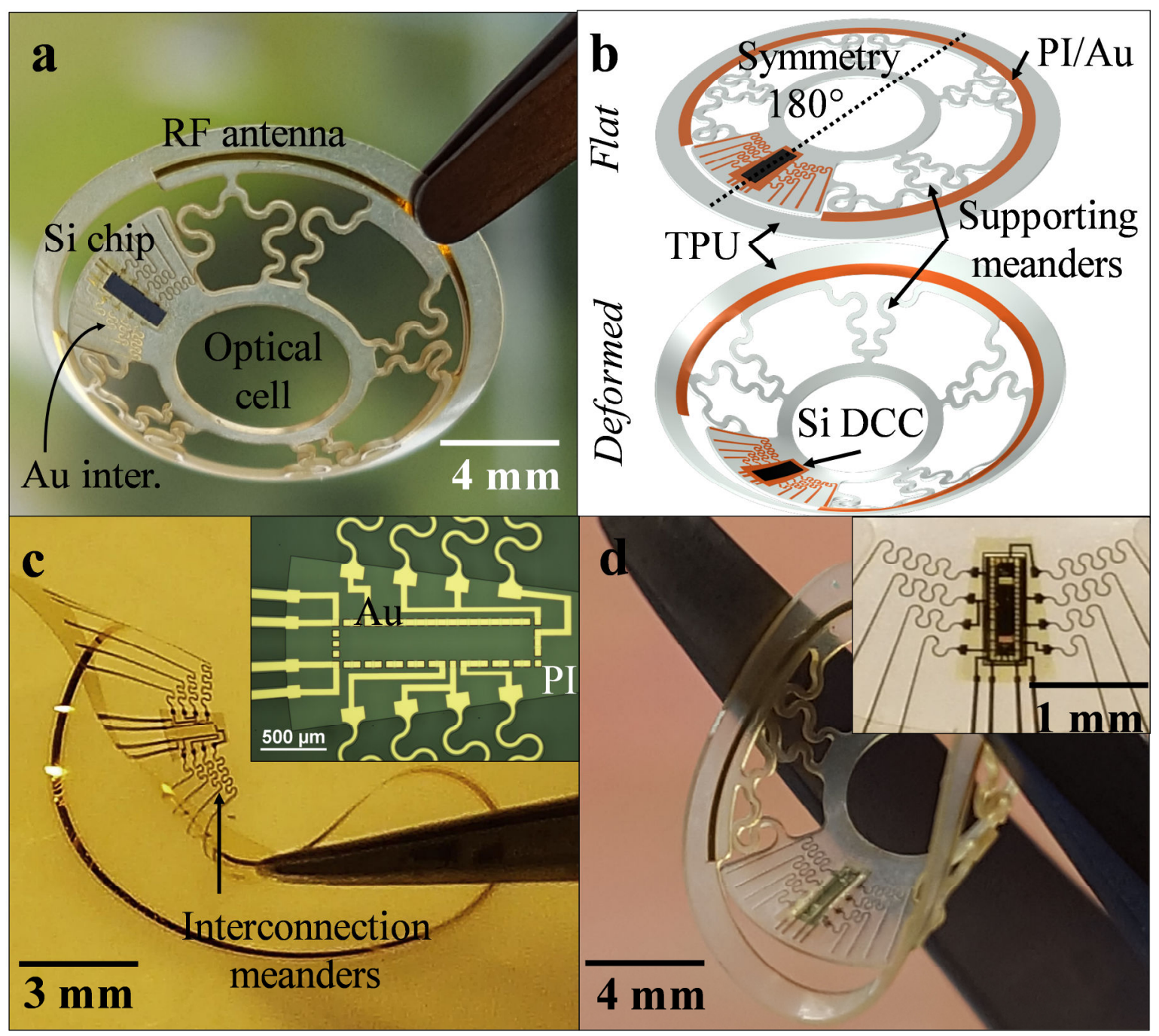

Figure 1. Schematic representation of the stretchable electronic platform. a) Picture of the thermoformed stretchable platform including Si chip RF antenna, thin-film interconnections and center region reserved for optical cells. b) Schematic representation of the thermoforming from a 2D plane to a 3D spherical-cap geometry. c) Self-standing stretchable thin-film circuitry based on a stack of polyimide/gold/polyimide. Rigid islands are connected by meanders to enable in-plane stretchability. d) Picture of a platform bended by a pair of tweezers, with a closed-up image of the Si chip. 


\section{WILEY-VCH}

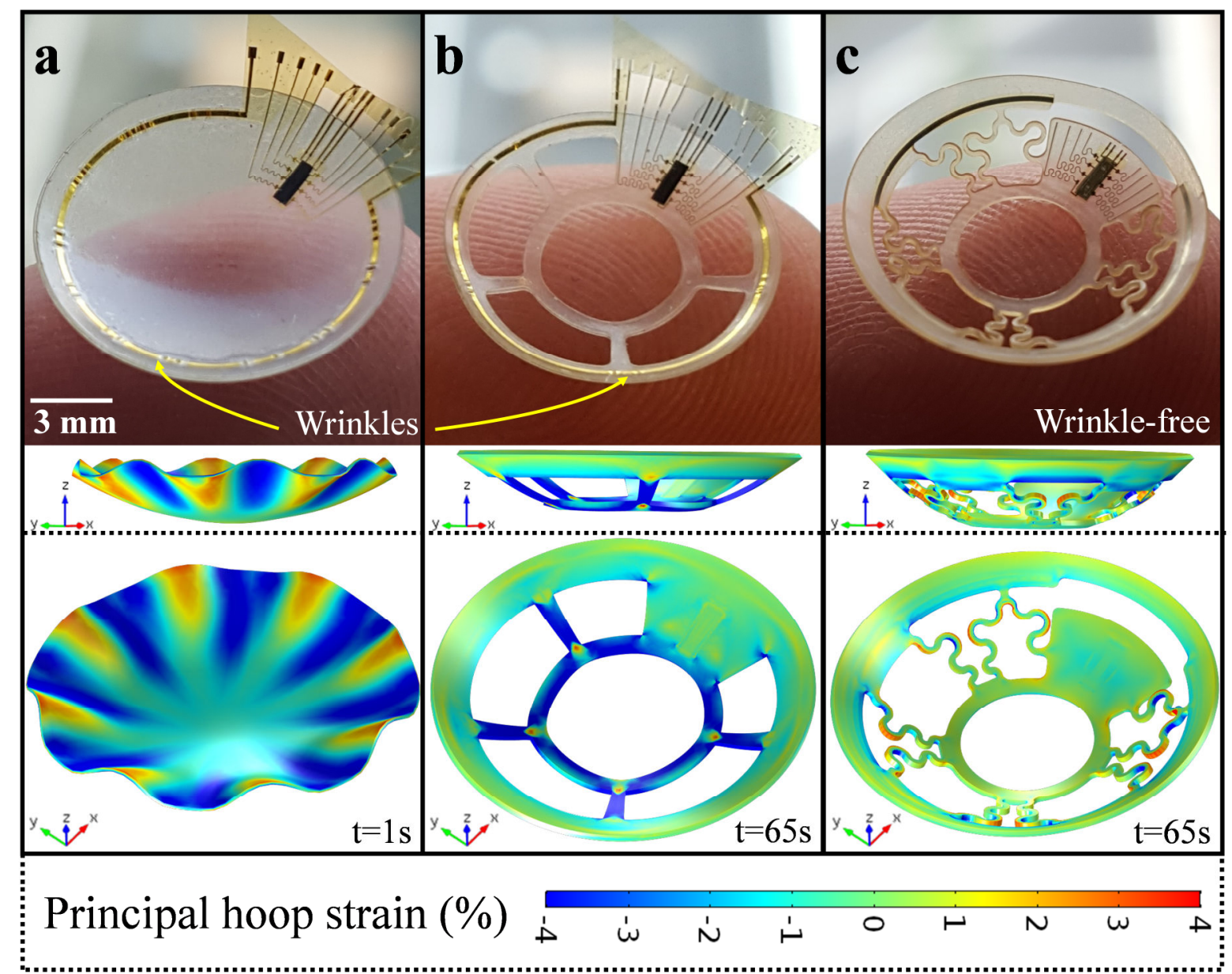

Figure 2. Pictures and simulated principal hoop strain of thermoformed TPU platforms with embedded thin-film circuits and DCC. a) TPU platform without patterns showing buckling near the outer edge which causes wrinkles along the RF antenna. The numerical simulation of a full disk predicts folds with localized strain at the start of the thermoforming process $(\mathrm{t}=1 \mathrm{~s})$. b) TPU platform with straight supports between inner and outer rings showing wrinkles near the outer edge and localized at the base of the supports. The inner and outer rings are distorted by the pulling action towards the center of the mold. c) Wrinkle-free TPU platform with meandered-shape supports. The numerical simulation predicts higher sag and closer curvature with respect to the mold. 


\section{WILEY-VCH}

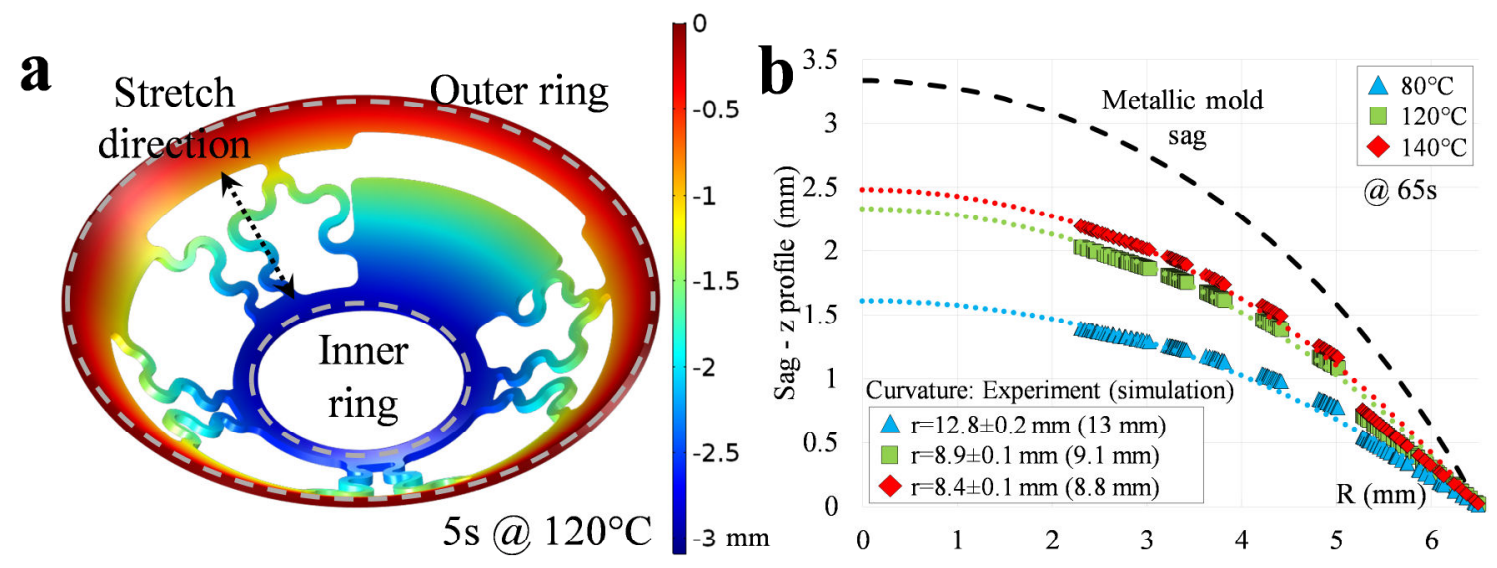

c Principal hoop stress $[\mathrm{MPa}]$

d Principal hoop strain (\%)
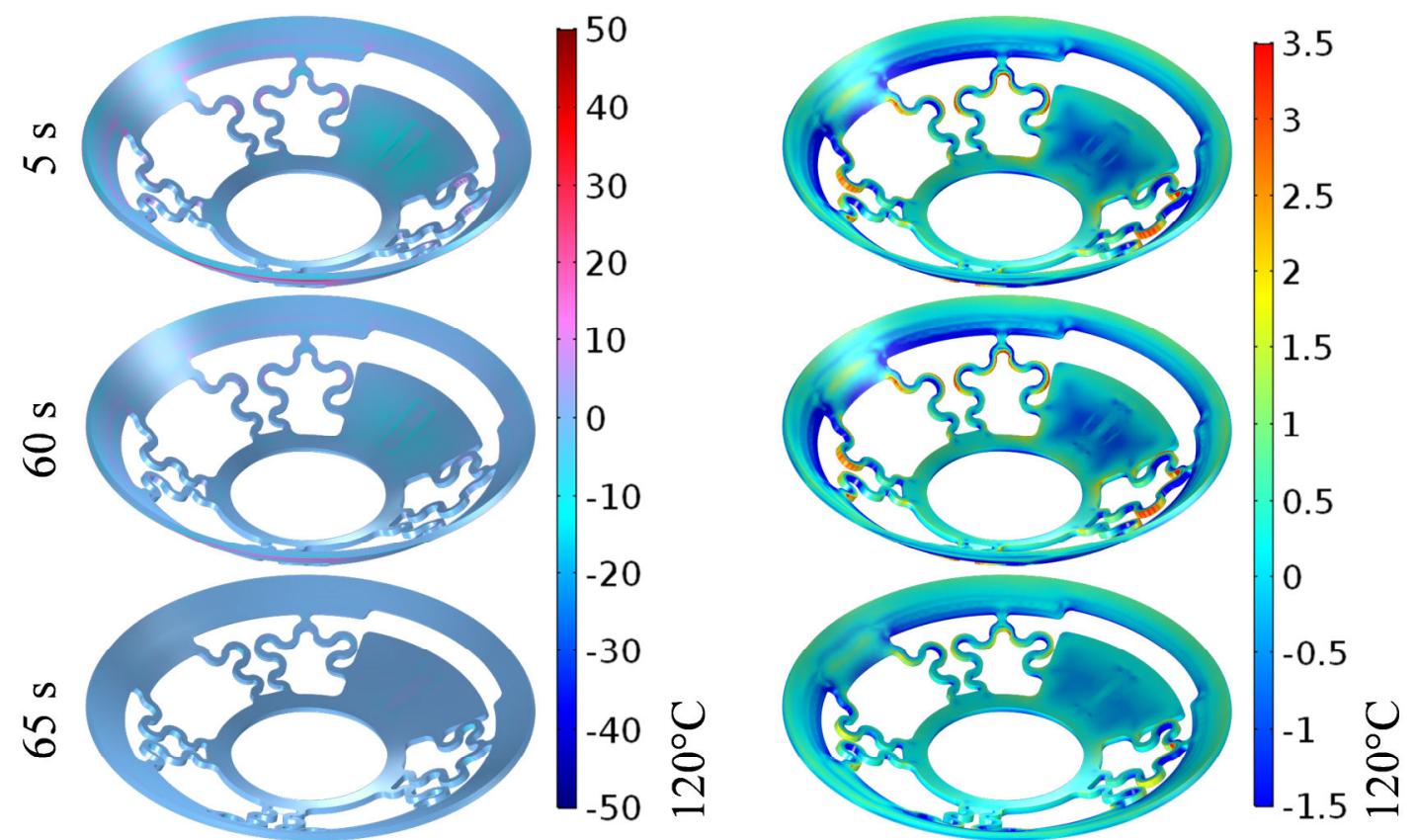

Figure 3. Mechanical analysis of the stretchable platform throughout the thermoforming process. a) Vertical displacement of the platform (including Si chip and interconnections) at the maximum deformation $\left(5 \mathrm{~s}\right.$ at $\left.120^{\circ} \mathrm{C}\right)$. b) Simulated and fitted curvature of the platform at different processing temperatures. c-d) $3 \mathrm{D}$ representation of the principal hoop stress and hoop strain at different thermoforming states, respectively. 


\section{WILEY-VCH}
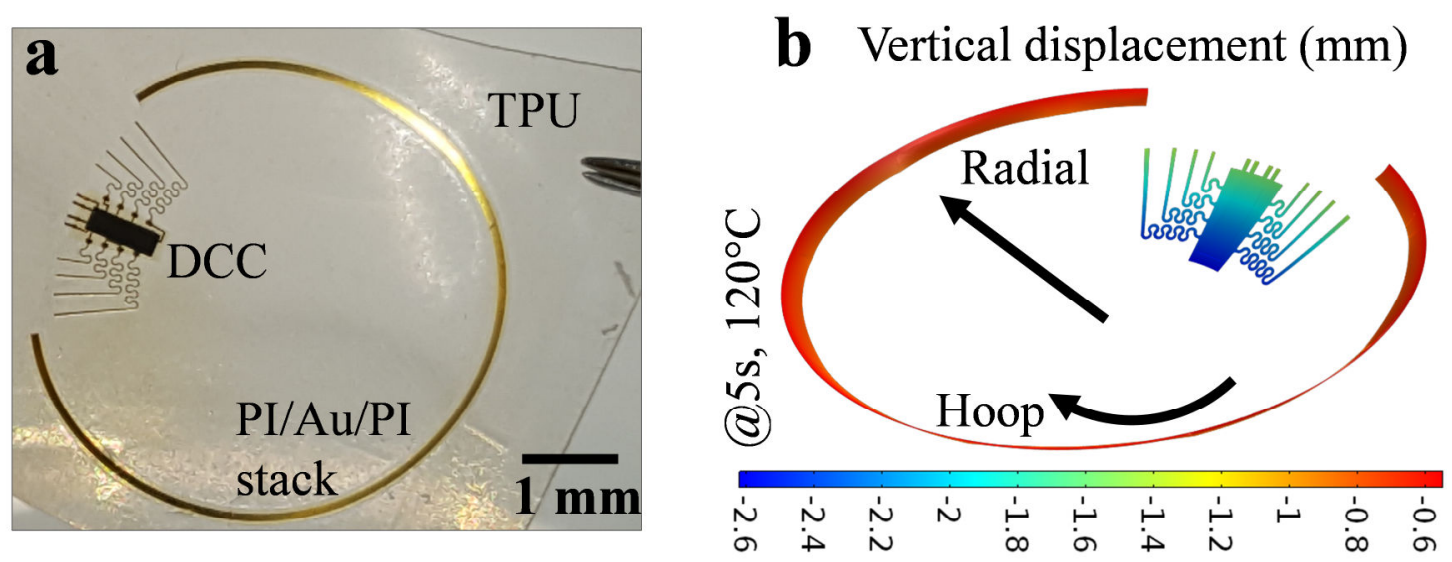

c Principal hoop strain (\%)
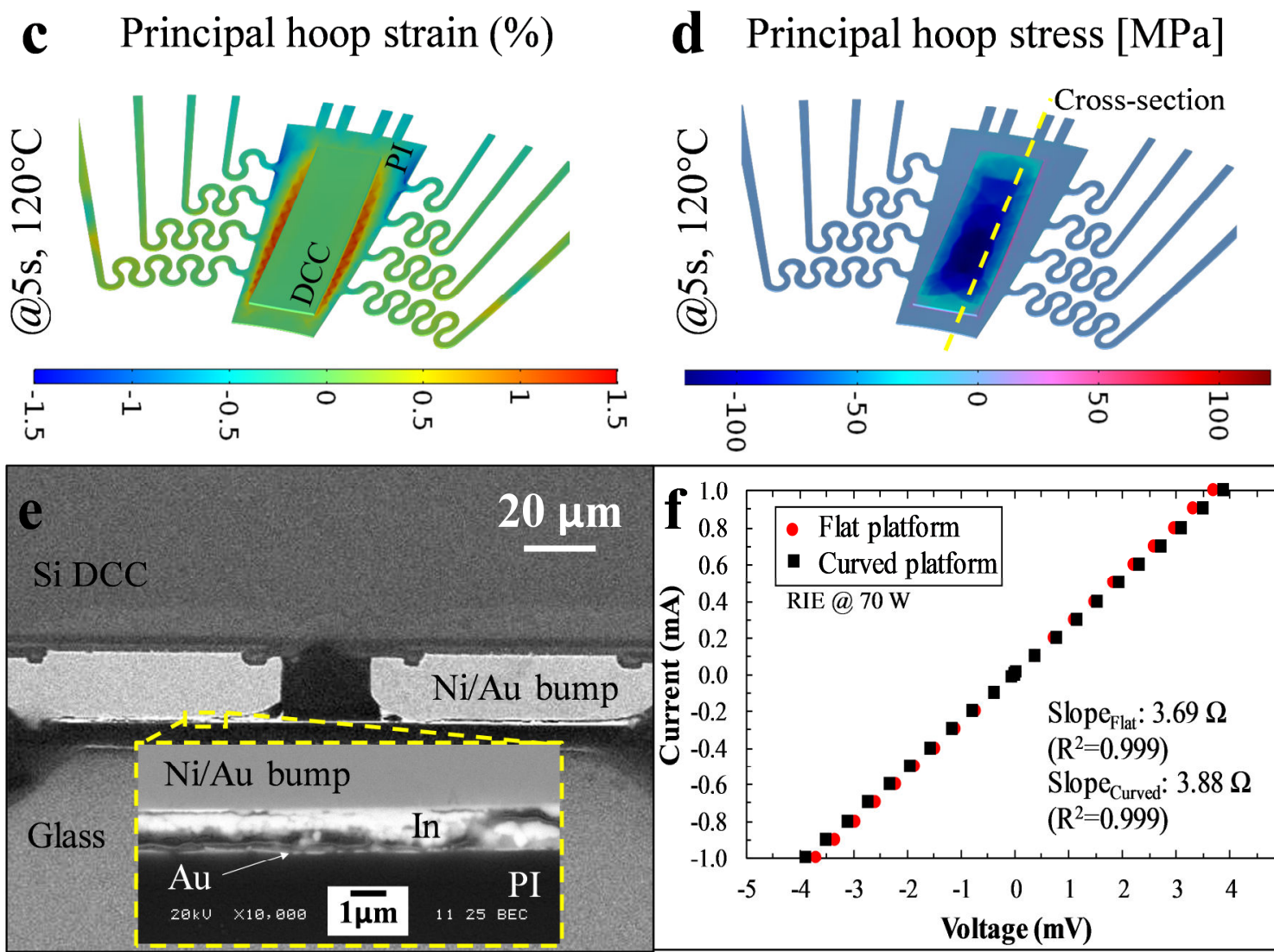

Figure 4. Mechanical analysis of the stretchable thin-film circuitry. a) Picture of the thin-film circuitry embedded on flat TPU substrates. b) Vertical displacement of the thin-film circuitry including Si chip and interconnections (aperture angle: $40^{\circ}$ ) at the maximum deformation. cd) 3D representation of the maximum principal strain and stress, respectively. e) SEM pictures of the stack's cross-section indicating the location of the indium/Au interface. $f$ ) Measured current versus voltage at the single vias $\left(R_{v l}\right)$ highlighted in $\mathrm{d}$, indicating a linear response thus ohmic contact before (red circles) and after (black squares) thermoforming. 


\section{WILEY-VCH}

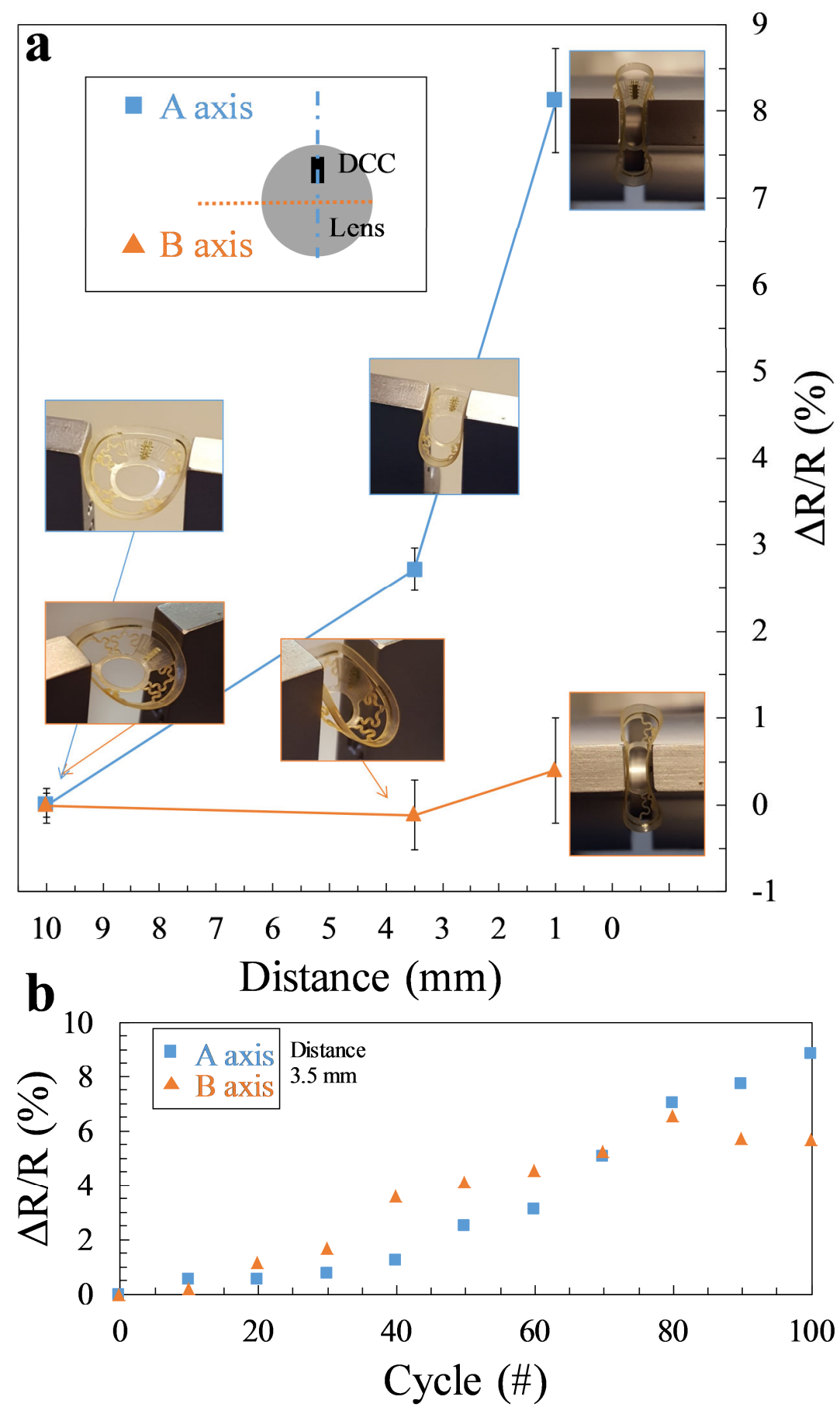

Figure 5. Results of the static and dynamic bending tests of the platforms. a) Measured relative change in resistance at different static deformation distances from $10 \mathrm{~mm}$ to $1 \mathrm{~mm}$ and along the A axis (along the DCC) and B axis (perpendicular to the DCC). b) Measured relative changes in resistance every 10 cycles of deformation at $3.5 \mathrm{~mm}$, along the $\mathrm{A}$ and $\mathrm{B}$ axis. 


\section{WILEY-VCH}
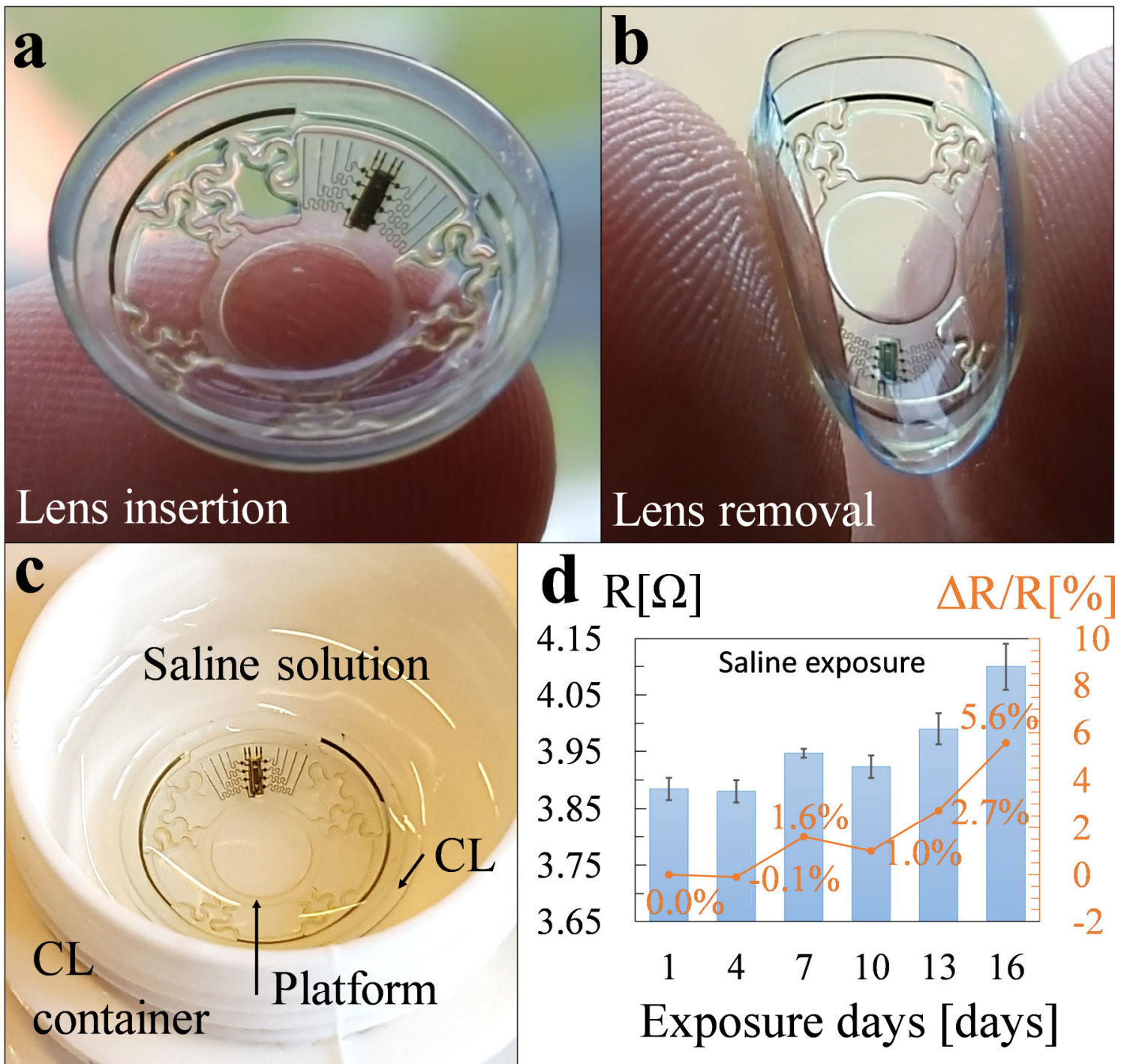

4.05

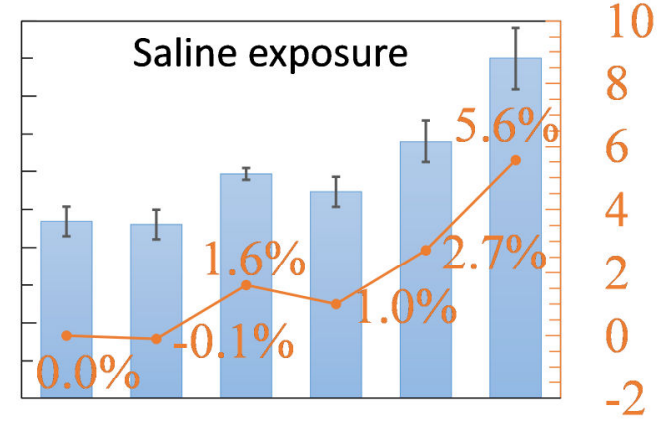

3.65

$\begin{array}{llllll}1 & 4 & 7 & 10 & 13 & 16\end{array}$ Exposure days [days]

Figure 6. Pictures of the stretchable platform when attached to a commercial soft contact lens, suggesting potential user modes. a) Picture of the stack on a finger's tip, mimicking insertion mode into the user's eye. b) Picture of the stack bended between finger's tips, mimicking the removal mode from the user's eye. c) The stack is submerged in a saline solution (used to keep the lens wet and clean), placed on a lens container. d) Measured resistance (standard deviation from three samples) (left axis) and relative change in resistance (right axis) with respect to exposure days in saline solution ( $0.9 \%$ saline), from 1 to 16 days. 


\section{WILEY-VCH}

We introduce a comprehensive approach to thermoform planar structures based on thermoplastic polyurethane (TPU) films into wrinkle-free spherical-cap platforms. Such platforms include thin-film circuits and silicon chips and are envisaged to be used for smart contact lens applications. Mechanical evolution throughout thermoforming was studied by numerical models in order to optimize strain-release patterns and increase the platform's stretchability.

Thermoplastic polyurethane (TPU); thermoforming; viscoelastic modelling; stretchable electronics; smart contact lens

Andrés Vásquez Quintero*, Rik Verplancke, Herbert De Smet and Jan Vanfleteren

Stretchable Electronic Platform for Soft and Smart Contact Lens Applications

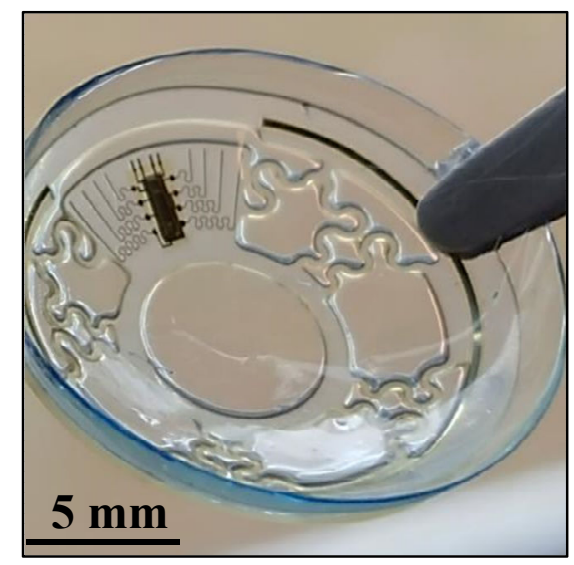




\section{WILEY-VCH}

Copyright WILEY-VCH Verlag GmbH \& Co. KGaA, 69469 Weinheim, Germany, 2013.

\section{Supporting Information}

\section{Stretchable Electronic Platform for Soft and Smart Contact Lens Applications}

Andrés Vásquez Quintero*, Rik Verplancke, Herbert De Smet and Jan Vanfleteren
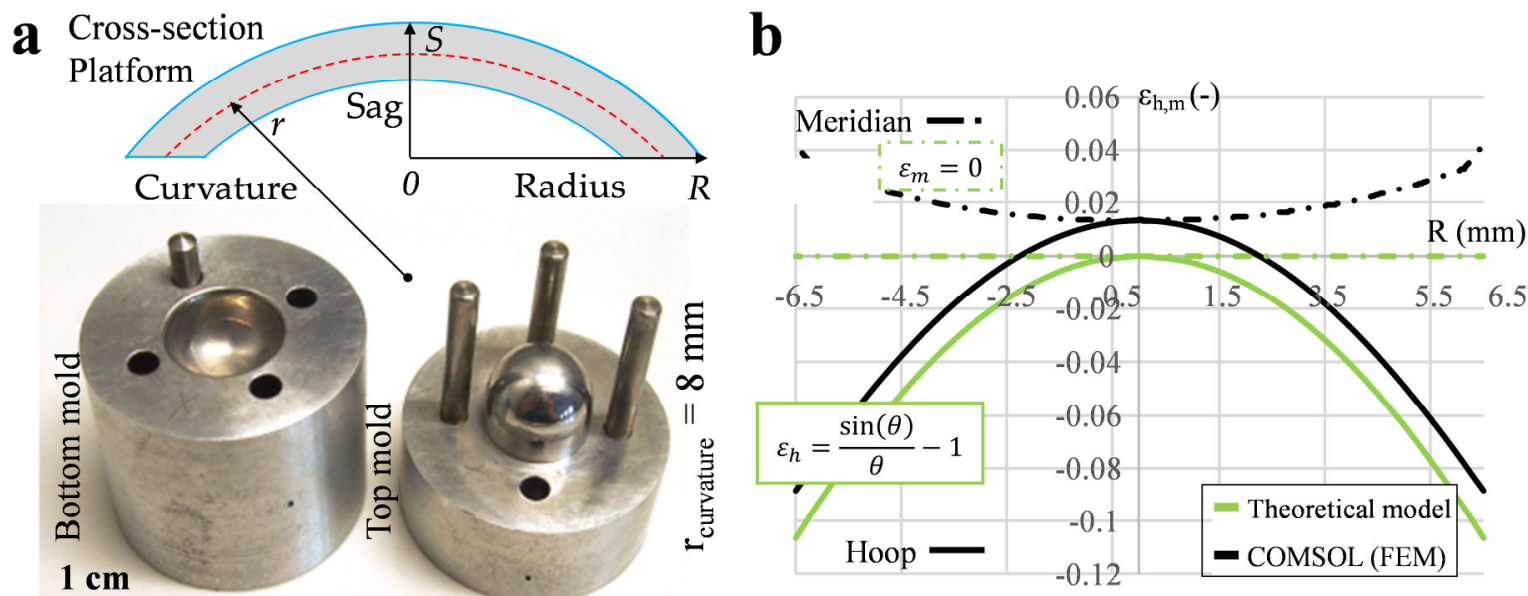

Figure S1. a) Cross-section of the platform indicating its dimensions as curvature radius ' $r$ ', maximum sag ' $S$ ' and initial disk radius ' $R$ '. Picture of the aluminum-based molds with a radius of curvature of $8 \mathrm{~mm}$ used to thermoform the stretchable platform at different temperatures. b) Calculated (equation in inset) ${ }^{[47]}$ and simulated (FEM) strain profiles along the meridian and hoop directions for a circular platform without pattern of $6.5 \mathrm{~mm}$ of disk radius. 


\section{WILEY-VCH}

$\mathbf{a}$

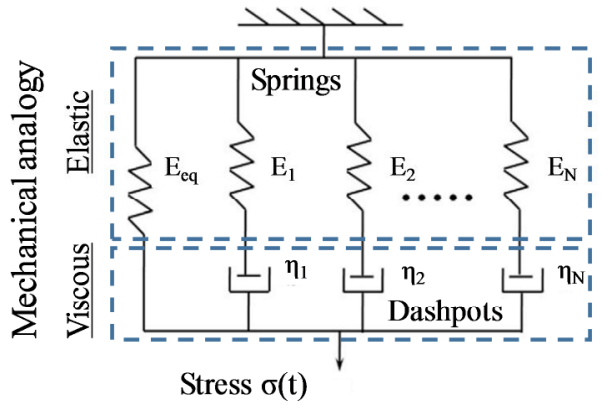

c

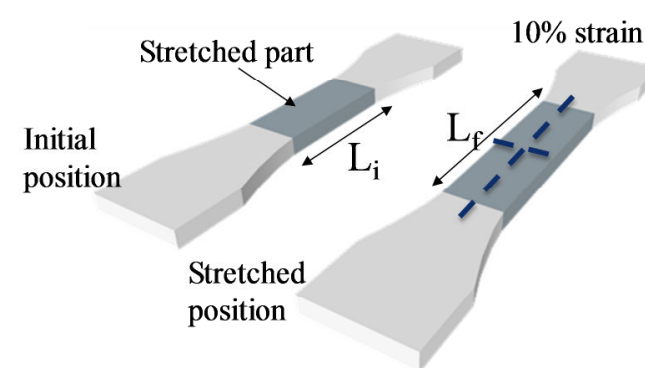

C

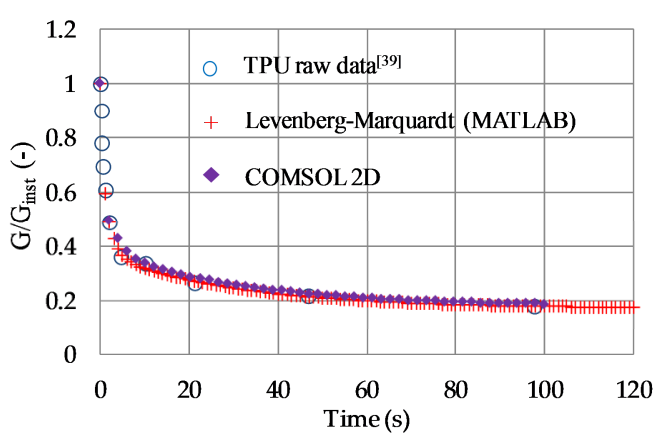

b Relaxation at constant deformation
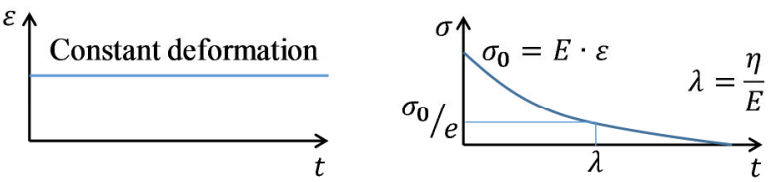

$\sigma(t)=\varepsilon_{0} E(t)+\int_{0}^{t} E(t-\xi) \frac{d \varepsilon(\xi)}{d \xi} d \xi$

d

2D double symmetry FEM model

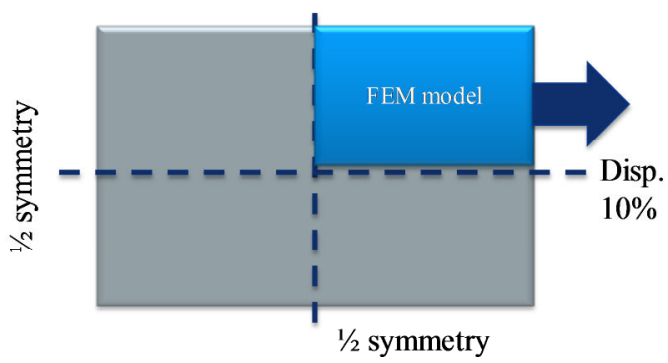

From L-M script

Coefficients for $\mathrm{N}=4$

$e_{1}=0.24, \lambda_{1}=0.22 \mathrm{~s} \quad e_{3}=0.20, \lambda_{3}=29.88$ $e_{2}=0.38, \lambda_{2}=1.80 \mathrm{~s} \quad e_{4}=0.0047, \lambda_{4}=1000 \mathrm{~s}$

$$
e(t)=1-e_{i} \sum_{1}^{N}\left(1-\exp \left(\frac{-t}{\lambda_{i}}\right)\right)
$$

Figure S2. Representation and validation of the Generalized Maxwell viscoelastic model. a) Mechanical analogy of the constitutive Maxwell model ${ }^{[48]}$ including springs and dashpots. b) Strain and stress profiles during the relaxation test used to extract the constitutive coefficients of the Maxwell model. c) Graphical representation of the relaxation test at $10 \%$ of strain. d) Double symmetry 2D geometry used to validate the fitted constitutive coefficients. e) Comparison between raw data, simulated data and fitted data of TPU relaxation tests. ${ }^{[53]}$ Relaxation tests of TPU at $10 \%$ deformation and $200{ }^{\circ} \mathrm{C}$ (above its glass transition temperature $)^{[53]}$ provide the experimental data to fit the relevant constitutive coefficients of the Maxwell viscoelastic model (i.e. $\epsilon$ and $\tau$ ). f) List of constitutive coefficients including the values for the normalized shear modulus $(e)$ and time $(\lambda)$. The coefficients list (four sets of constitutive coefficients resulted in an adequate compromise between resolution in time and calculation power), were fitted with the Levenberg-Marquardt algorithm. The WilliamLandel-Ferry thermal shift function is: $E^{T}(t)=E^{T_{0}}(t / A(T)), \log (A(T))=-\frac{C_{1}\left(T-T_{0}\right)}{C_{2}+\left(T-T_{0}\right)}$, where $E^{T}$ is the temperature dependent modulus, $T_{0}$ is the reference temperature (normally selected as the glass transition temperature of the modelled material), $T$ is the actual temperature, $A(T)$ is the time reduction factor, $t$ is the actual time, and $C_{1}$ and $C_{2}$ are two empiric constants at the reference temperature, respectively. For the material used here (Platilon ${ }^{\circledR} \mathrm{U} 4201$ AU from BAYER ${ }^{\circledR}$ ) these constants are suggested as $C_{l}=3$ and $C_{2}=37.64{ }^{\circ} \mathrm{C} .{ }^{[50,53]}$ 


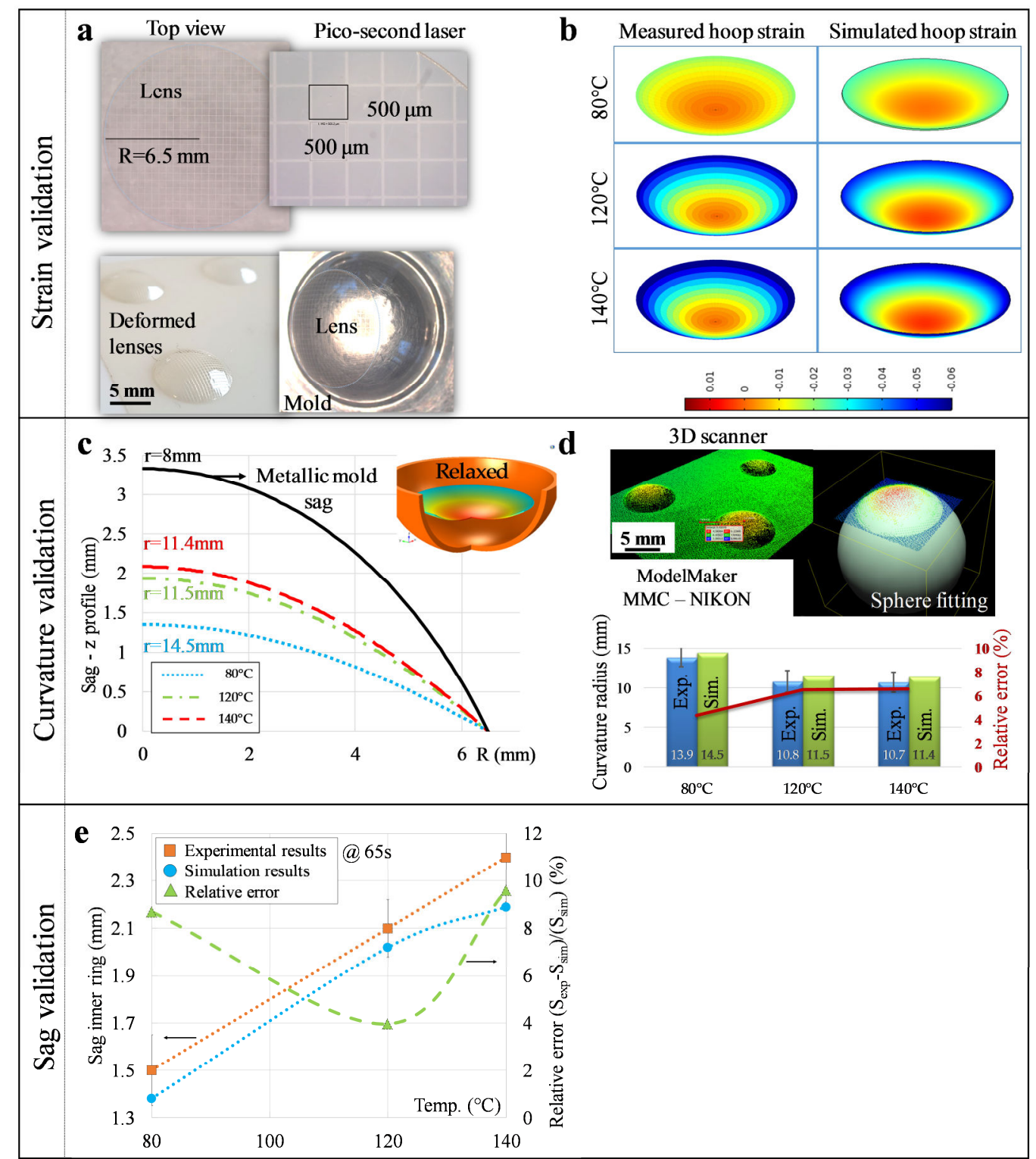

Figure S3. Validation procedure and results of the 2D thermoforming FEM model using a full circle (without pattern). a) Laser-ablated grid (500 $\mu \mathrm{m} \times 500 \mu \mathrm{m}, 5 \mu \mathrm{m}$ deep) used to measure the meridian and hoop strain on the platforms before and after the thermoforming. The grid on the thermoformed platforms is measured on top of a spherical mold in order to have perpendicular view from the top. b) Comparison of measured (using the grid) and simulated (2D FEM) hoop strain at different processing temperatures. c. Results of the curvature FEM simulation at different temperatures $80^{\circ} \mathrm{C}, 120^{\circ} \mathrm{C}$ and $140^{\circ} \mathrm{C}$, represented with a dotted blue line, a dotted-stripe line and striped line, respectively. The solid black line represents the sag of the metallic mold along the radial direction. d) Image of the data cloud set from the model maker MMC-NIKON. The latter is used to scan the top surface of the TPU disks with a precision of $12 \mu \mathrm{m}$. Also, relative errors between measured (using the grid) and simulated data are presented with values lower than $6 \%$. e) Simulated and measured sag of the platform's inner ring at different processing temperatures at the relaxed state $(65 \mathrm{~s})$. The relative error is displayed on the right hand side. The relative error reaches a minimum $(4 \%)$ at $120^{\circ} \mathrm{C}$, just above the TPU glass transition temperature $\left(T_{g} \sim 100{ }^{\circ} \mathrm{C}\right)$, where the viscoelastic model is expected to be more accurate due to its definition. 


\section{WILEY-VCH}
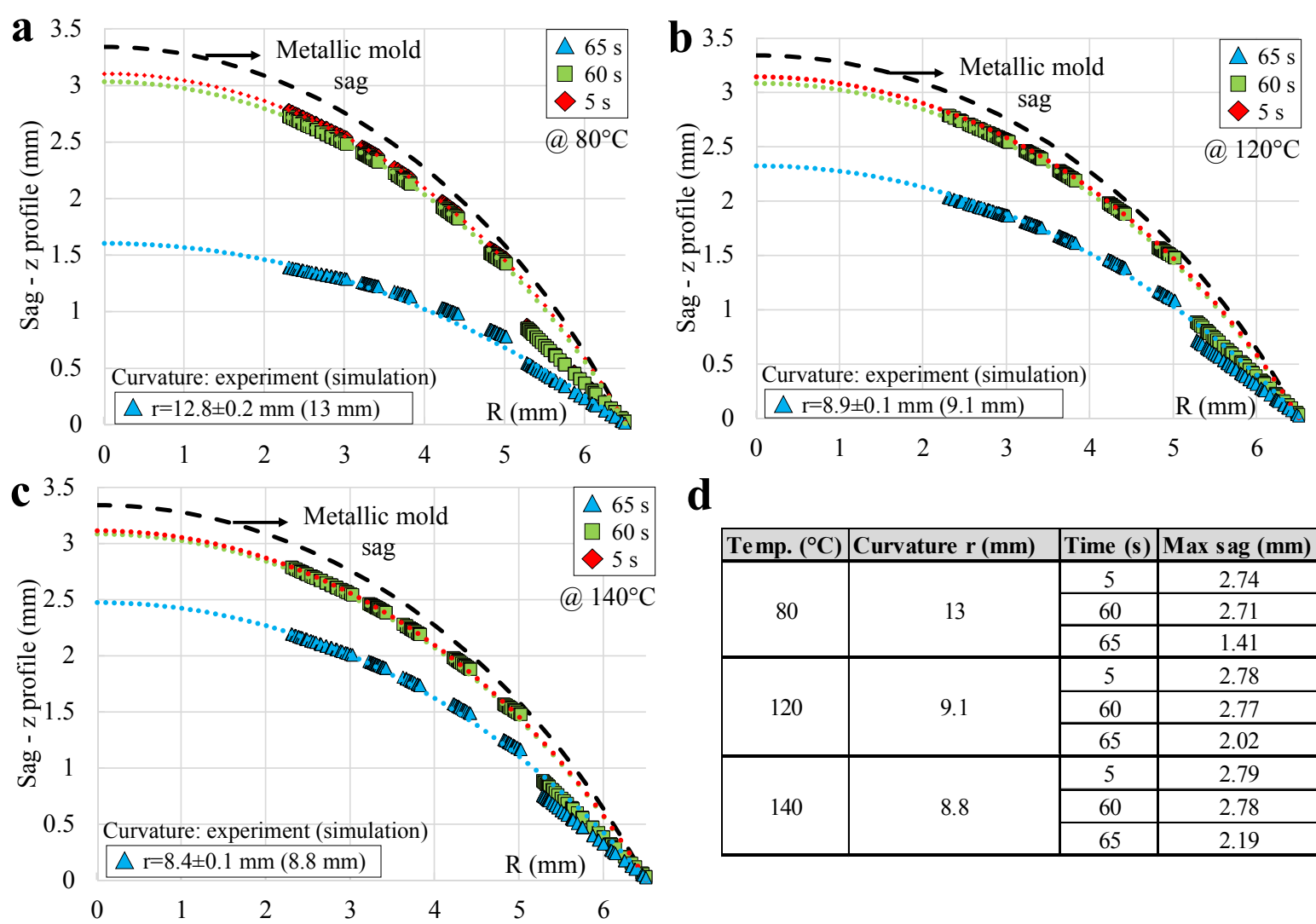

d

\begin{tabular}{|c|c|c|c|}
\hline Te mp. $\left({ }^{\circ} \mathbf{C}\right)$ & Curvature $\mathbf{r}(\mathbf{m m})$ & Time $(\mathbf{s})$ & Max sag $(\mathbf{m m})$ \\
\hline \multirow{3}{*}{80} & \multirow{3}{*}{13} & 5 & 2.74 \\
\cline { 3 - 4 } & & 60 & 2.71 \\
\hline \multirow{3}{*}{120} & \multirow{3}{*}{9.1} & 65 & 1.41 \\
\hline \multirow{3}{*}{140} & & 5 & 2.78 \\
\hline \multirow{3}{*}{} & \multirow{3}{*}{8.8} & 60 & 2.77 \\
\cline { 3 - 4 } & & 65 & 2.02 \\
\hline & & 5 & 2.79 \\
\cline { 3 - 4 } & & 60 & 2.78 \\
\hline
\end{tabular}

Figure S4. Simulated and fitted curvature of the stretchable platforms at different states of the thermoforming and at different processing temperature along the radial direction, where $0 \mathrm{~mm}$ corresponds to the center of the platform. a) Sag ' $S$ ' at $80^{\circ} \mathrm{C}$. b) Sag ' $S$ ' at $120^{\circ} \mathrm{C}$. c) Sag ' $S$ ' at $140^{\circ} \mathrm{C}$. Simulated results at $5 \mathrm{~s}, 60 \mathrm{~s}$ and $65 \mathrm{~s}$ represented with red diamonds, green squares and blue triangles, respectively. d) Summary table including the platform's radius of curvature ' $r$ ' and maximum sag ' $S$ ' at different states of the thermoforming. 


\section{WILEY-VCH}
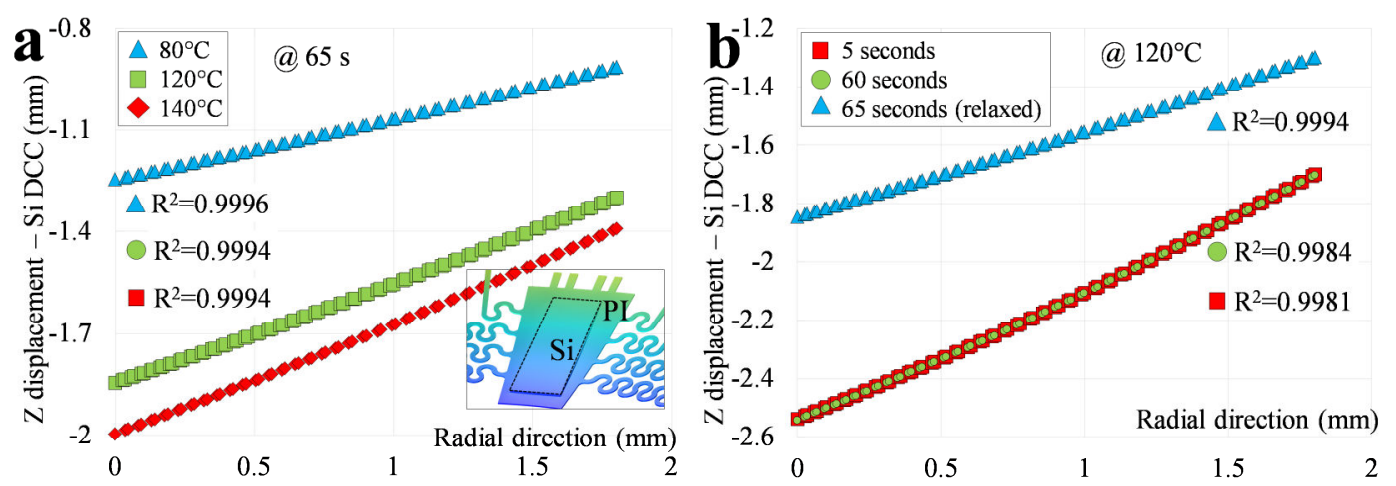

C
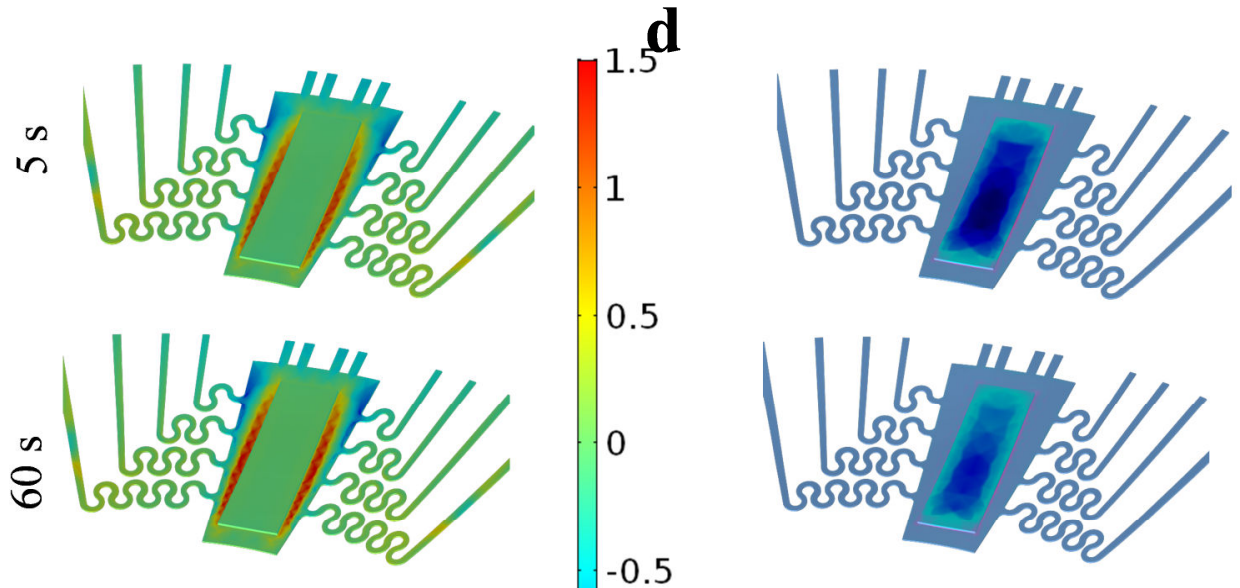

0.5
0
-0.5
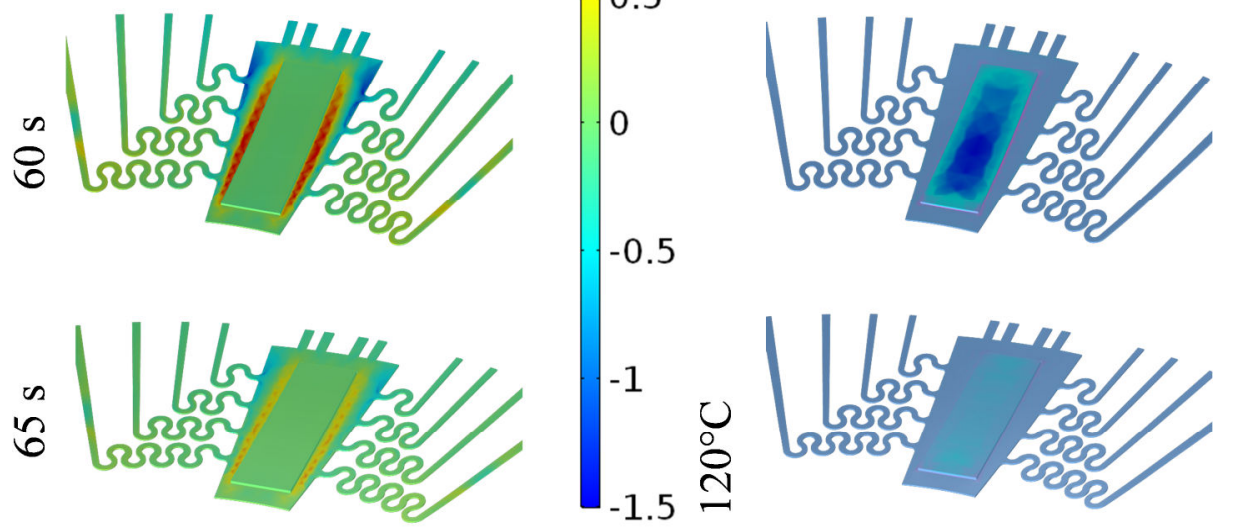

Figure S5. Mechanical analysis of the DCC curvature and meanders deformation. $a-b)$ Linearity of the thinned-down Si chip after thermoforming at different processing temperatures and the relaxed state $(65 \mathrm{~s})$, and at different states of the thermoforming at $120^{\circ} \mathrm{C}$. The coefficients of determination ' $R$ ' ' are shown for each case. A displacement of zero mm corresponds to the initial flat position of the DCC. The flatness of the chip is maintained at different processing temperatures (i.e. 80,120 and $140^{\circ} \mathrm{C}$ ) with a relatively high linearity (coefficient of determination $\left.\mathrm{R}^{2}=0.9994\right)$. At a fixed temperature $\left(120^{\circ} \mathrm{C}\right)$ the evolution of the DCC vertical location throughout the thermoforming is shown in Figure $b$. At the maximum deformation $(5 \mathrm{~s})$ the DCC has a curvature radius of $9.1 \mathrm{~mm}$, however, at the relaxed state $(65 \mathrm{~s})$ it recovers its flatness protecting its mechanical integrity. c-d) 3D representation of the principal stress and strain values at different thermoforming states and $120^{\circ} \mathrm{C}$, respectively. 


\section{WILEY-VCH}
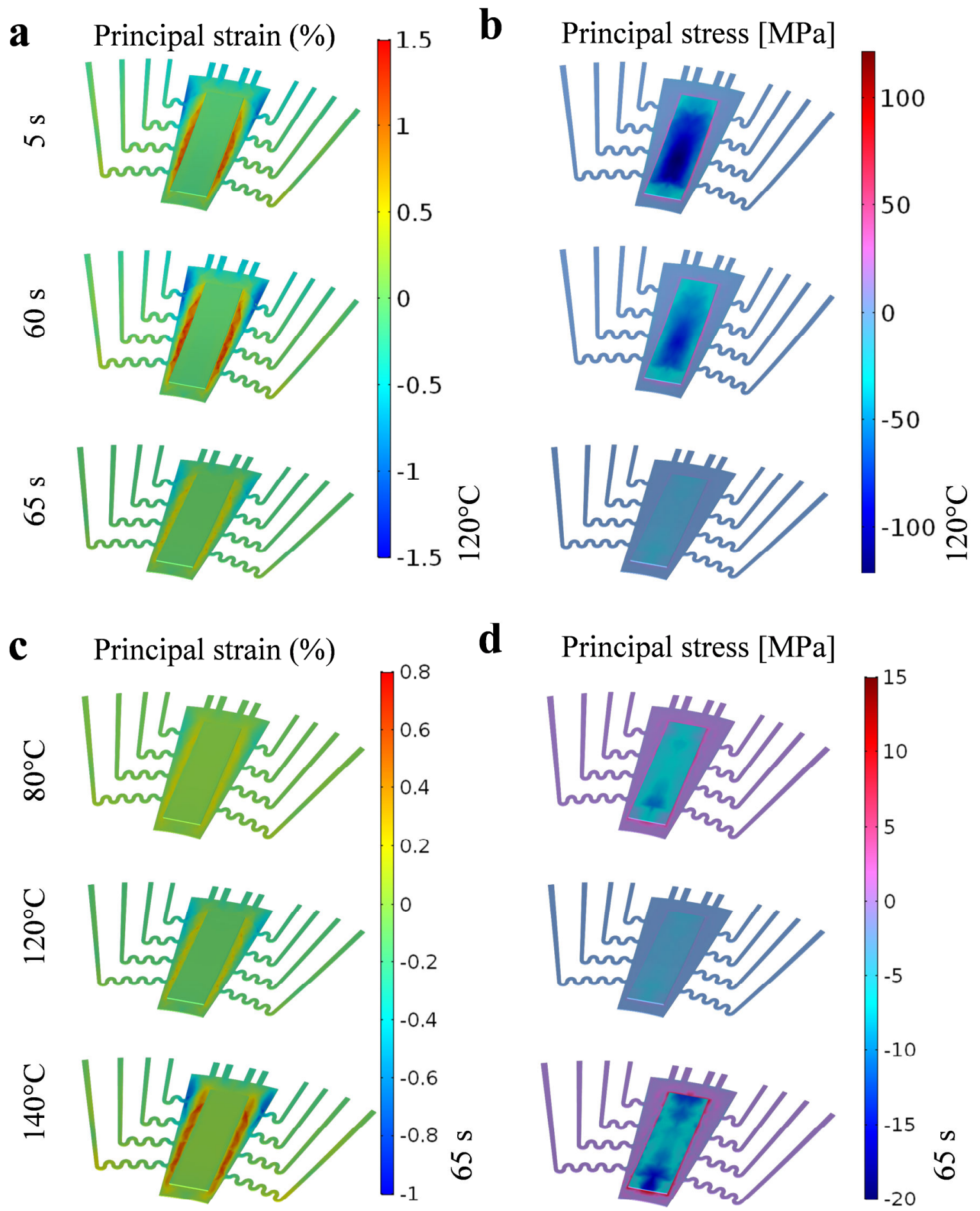

d
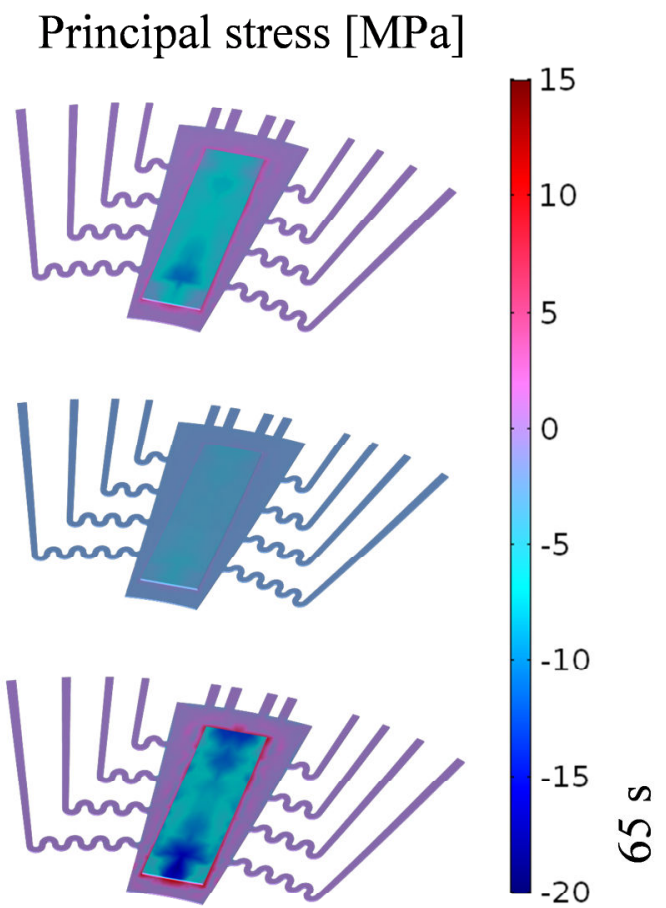

Figure S6. 3D representation of the strain and stress distributions at the DCC chips and meandered-interconnections (aperture angle of $0^{\circ}$ ) at different states of the thermoforming and processing temperatures. a-b) Principal strain and stress distributions at different states and at $120^{\circ} \mathrm{C}$, respectively. c-d) Principal strain and stress distributions at the relaxed state (65 s) and different processing temperatures, respectively. 


\section{WILEY-VCH}

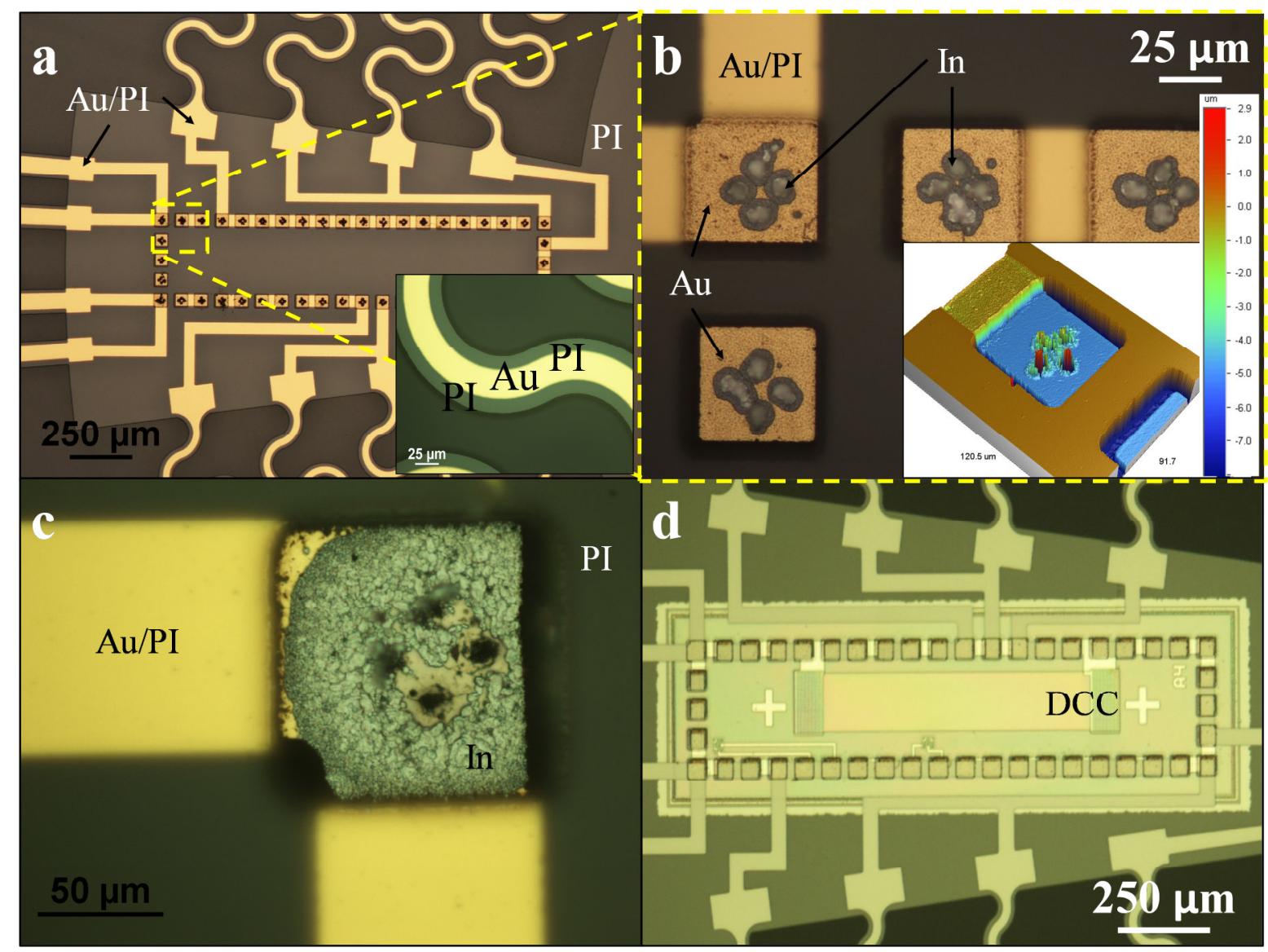

Figure S7. Characterization of the LIFTed indium bumps. The LIFT technique allows transferring a controllable amount of material with a relatively high precision and high yield.$^{[42,54]}$ Three spatially coincident bumps of In $(10 \mu \mathrm{m}$ wide and $7 \mu \mathrm{m}$ thick $)$ enable the interconnection at a high pitch resolution without short circuits and/or contamination. a) Topview picture of the Au circuitry supported by polyimide at the location of the DCC. The mechanical robustness of the traces with respect to bending/stretching due to lateral forces was ensured thanks to an extra $25 \%$ of PI at each side, ${ }^{[5,56]}$ and by positioning them at the mechanical neutral plane. ${ }^{[43]}$ The meanders were designed according to maximum strain levels encountered during fabrication and/or daily lens usage (e.g. inserting into the eye), for instance, by modifying the trace width and aperture angle, etc. ${ }^{[57-59]}$ The present study analyzes two aperture angles for the interconnection meanders, being $0^{\circ}$ and $40^{\circ}$. b) Picture of the pad openings through the PI top layer showing the LIFT of indium profile measured with white light interferometer. c) Picture of one pad opening after the indium has been melted at $120{ }^{\circ} \mathrm{C}$ for 5 min. d) Bottom-view picture of the integrated DCC. 


\section{WILEY-VCH}

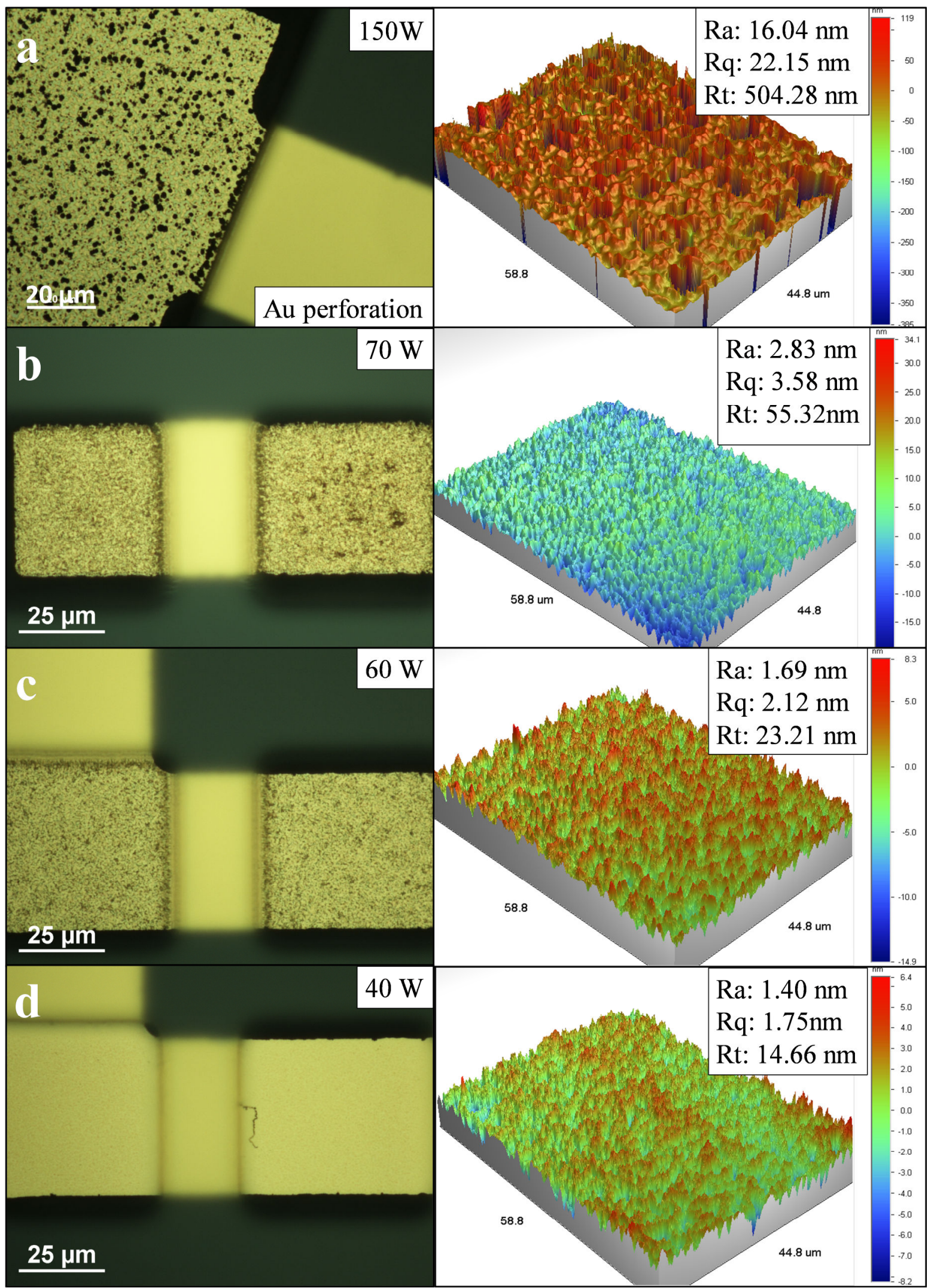

Figure S8. Optical images and white light interferometer images of the Au pads after opening with RIE technique at different etching powers. a) Au surface after RIE at 150W showing perforation and RMS roughness of $22.15 \mathrm{~nm}$. b) Au surface after RIE at $70 \mathrm{~W}$ with RMS roughness of $3.58 \mathrm{~nm}$. c) Au surface after RIE at $60 \mathrm{~W}$ with RMS roughness of $2.12 \mathrm{~nm}$. d) $\mathrm{Au}$ surface after RIE at 40W with RMS roughness of $1.75 \mathrm{~nm}$. 


\section{WILEY-VCH}
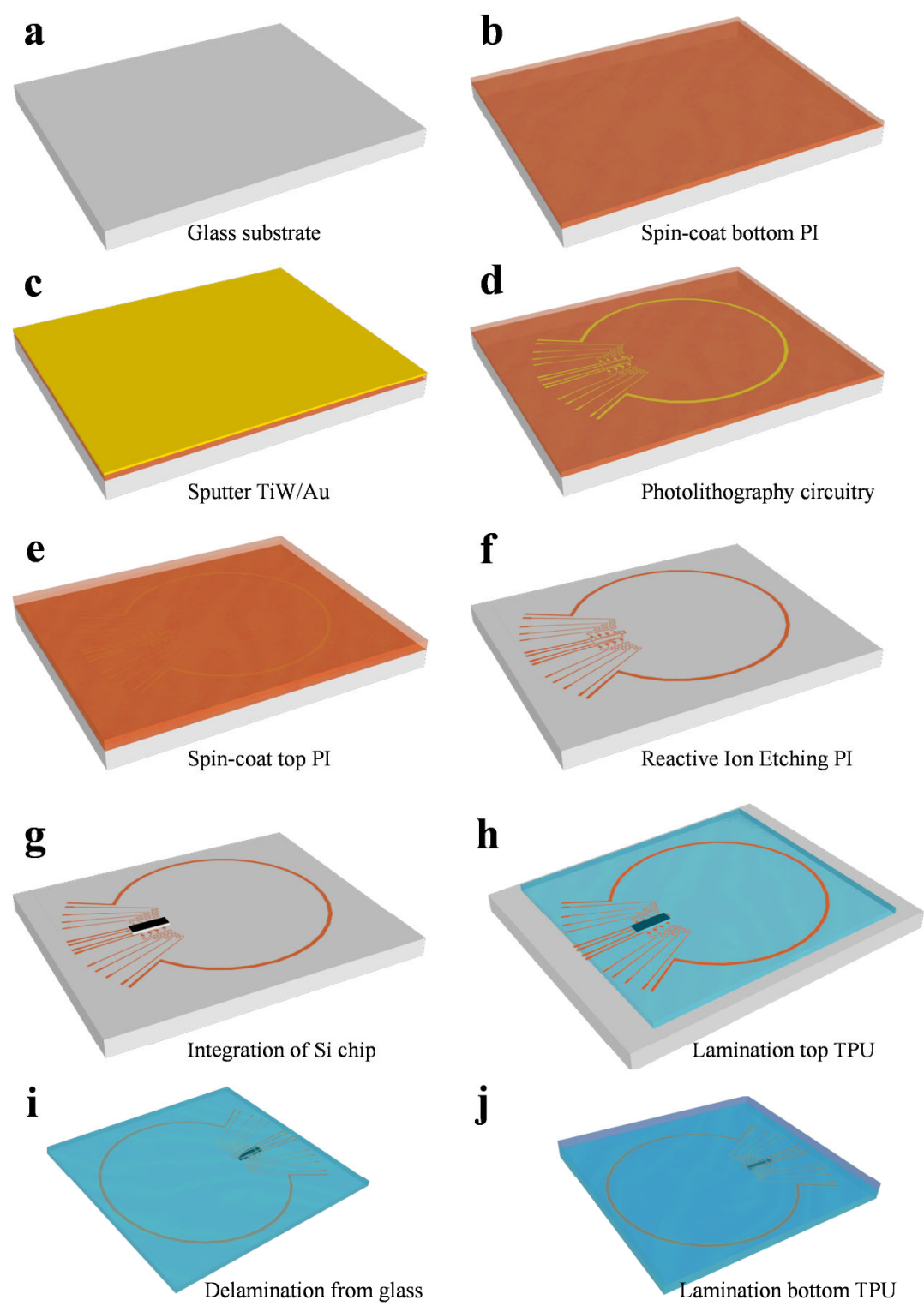

j
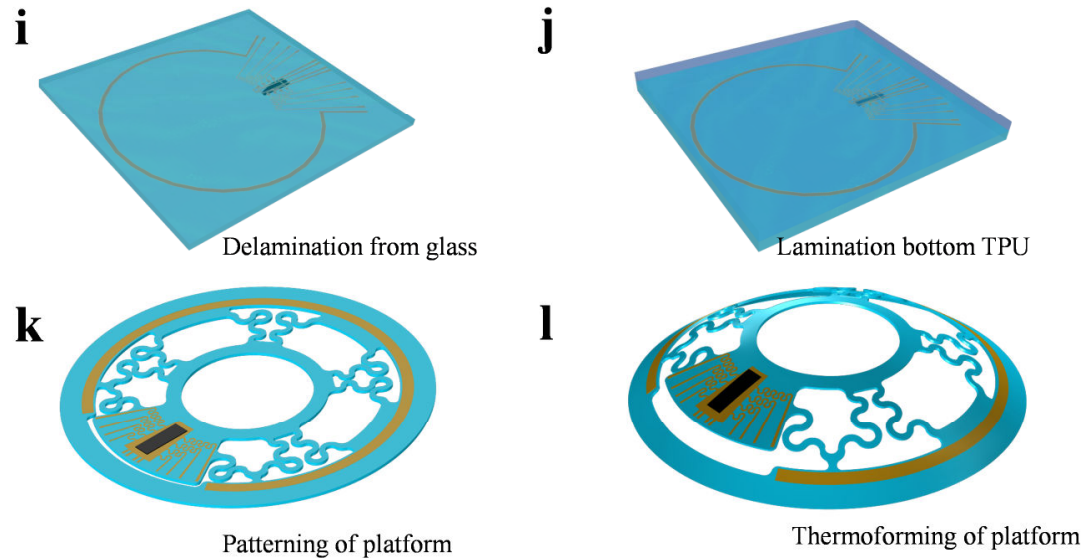

Figure S9. Processing steps of the deformable platform's fabrication. a) Cleaning of glass carrier substrate. b) Spin coating of the bottom PI layer (PI-2611). c) Sputtering of TiW/Au $(50 / 150 \mathrm{~nm})$. d) Photolithography and etching of Au circuitry. e) Spin coating of the top PI layer (PI-2611). f) Reactive Ion Etching of PI after sputtering and photolithography of TiW/Al hard mask (20 / $200 \mathrm{~nm})$. g) LIFT of indium and flip-chip integration of the DCC (5 min at $\left.120^{\circ} \mathrm{C}\right)$. h) Lamination of the top TPU layer $\left(10 \mathrm{~min}\right.$ at $\left.120^{\circ} \mathrm{C}\right)$. i. Delamination of the platform from the glass carrier. j. Lamination of the bottom TPU layer $\left(10 \mathrm{~min}\right.$ at $\left.120^{\circ} \mathrm{C}\right) \mathrm{k}$. Laser ablation of the TPU platform (both layers at the same time). 1. Thermoforming of the platform using metallic molds $\left(5 \mathrm{~min}\right.$ at $\left.120^{\circ} \mathrm{C}\right)$. 


\section{WILEY-VCH}
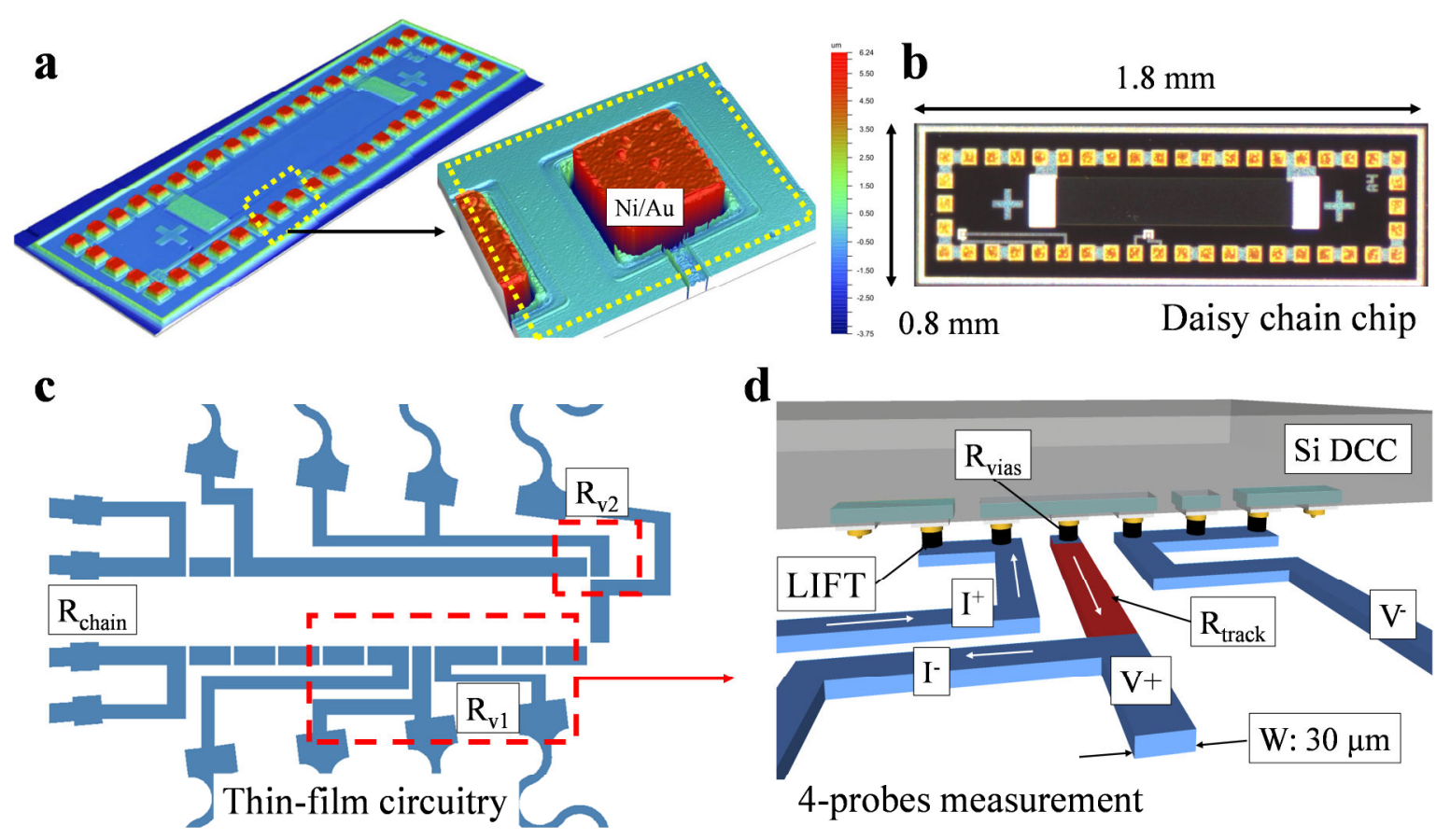

Figure S10. Layout of the daisy chain chip (DCC) and the thin-film circuitry used to validate the LITF-based integration technique. a) White light interferometer images of the DCC illustrating the height of the electroless plated Ni/Au bumps of $15 \mu \mathrm{m}$ above the chip surface. b) Optical image of the DCC with lateral dimensions. c) Layout of the thin-film Au circuitry used to test the interconnection of the DCC. Red dashed squares show the location of the vias measurement. d) Schematic of the 4-probes layout implemented to measure the resistance at $R_{v l}$. The red track indicates the resistance being measured in series to the vias.

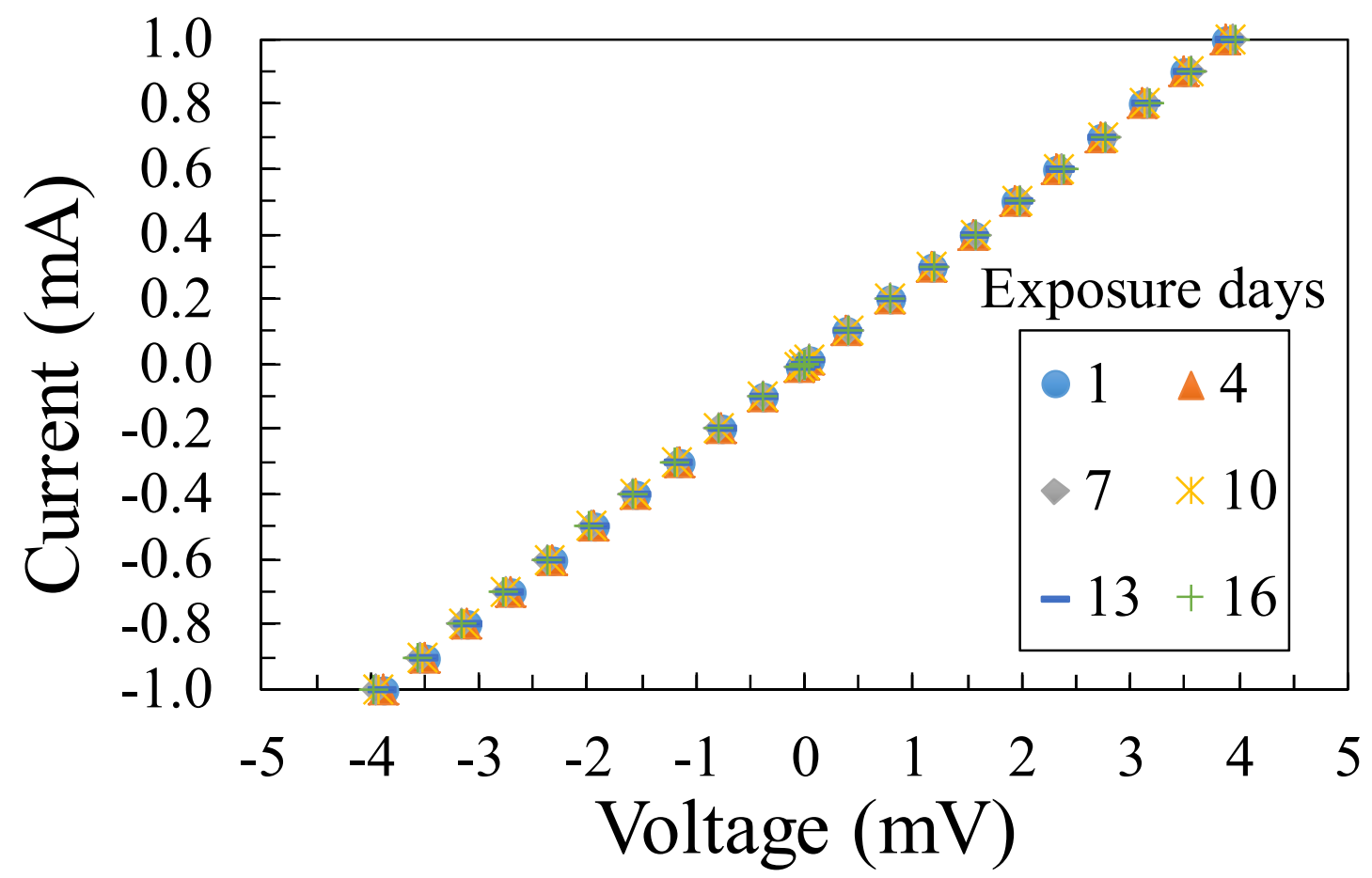




\section{WILEY-VCH}

Figure S11. Measured current versus voltage at a single vias of the DCC after several days (from 1 to 16) of immersion in the saline solution. The resistances presented in Figure 5d were calculated from the slope of the plot V/I. The linear response indicated an ohmic contact. 